\title{
8-oxoguanine DNA glycosylase1 recognizes oxidatively-generated epitranscriptomic marks on nascent mRNAs to promote RSV replication
}

\section{Ke Wang}

Northeast Normal University

\section{Wenjing Hao}

Chinese Academy of Sciences

\section{Lang Pan}

University of Texas Medical Branch at Galveston

\section{Zheng Xu}

School of Life Science, Northeast Normal University

\section{Attila Bácsi}

University of Debrecen, Hungary https://orcid.org/0000-0002-2311-2975

\section{Matthieu Gagnon}

University of Texas Medical Branch https://orcid.org/0000-0003-4516-604X

\section{Basu Ritwika}

University of Texas Medical Branch at Galveston

\section{Bing Tian}

University of Texas Medical Branch

\section{Radak Zsolt}

University of Physical Education

\section{Spiros Vlahopoulos}

National and Kapodistrian, University of Athens

\section{Tapas Hazra}

The University of Texas Medical Branch at Galveston

\section{Antonella Casola}

University of Texas Medical Branch at Galveston

\section{Roberto Garofalo}

University of Texas Medical Branch at Galveston

\section{Xueqing Ba}

Northeast Normal University https://orcid.org/0000-0002-1075-5496

\section{Allan Brasier}

University of Wisconsin-Madison https://orcid.org/0000-0002-5012-4090

Istvan Boldogh ( $\nabla$ sboldogh@utmb.edu ) 


\section{Article}

Keywords: respiratory syncytial virus (RSV) infection, microbiology, virology, epigenetics

Posted Date: May 20th, 2021

DOl: https://doi.org/10.21203/rs.3.rs-515369/v1

License: (c) (i) This work is licensed under a Creative Commons Attribution 4.0 International License. Read Full License 


\section{8-oxoguanine DNA glycosylase1 recognizes oxidatively-generated epitranscriptomic marks on nascent mRNAs to promote RSV replication}

Ke Wang ${ }^{1, a}$, Wenjing Hao ${ }^{1, b}$, Lang Pan ${ }^{1}$, Xu Zheng ${ }^{1, a}$, Matthieu G. Gagnon ${ }^{1,3,4}$, Ritwika Basu ${ }^{1}$, Bing Tian $^{3}$, Attila Bacsi ${ }^{1, \mathrm{c}}$, Zsolt Radak ${ }^{1, \mathrm{~d}}$, Spiros Vlahopoulos ${ }^{1, \mathrm{e}}$, Tapas K. Hazra ${ }^{3}$, Antonella Casola ${ }^{2}$, Roberto Garofalo², Xueqing Ba Allan R. Brasier ${ }^{5}$, and Istvan Boldogh ${ }^{1, *}$

Departments of ${ }^{1}$ Microbiology and Immunology, ${ }^{2}$ Pediatrics, ${ }^{3}$ Internal Medicine, ${ }^{3}$ Biochemistry and Molecular Biology, ${ }^{4}$ Sealy Center for Structural Biology and Molecular Biophysics, University of Texas Medical Branch at Galveston, Galveston, TX77555, USA; ${ }^{4}$ Key Laboratory of Molecular Epigenetics of Ministry of Education, School of Life Science, Northeast Normal University, Changchun, Jilin, China; ${ }^{5}$ nstitute for Clinical and Translational Research, University of Wisconsin-Madison School of Medicine and Public Health, Madison, Wisconsin 53705, USA

Author contributions: K.W, W.H, and I.B designed, performed, analyzed cell culture and animal experiments. L.P, X.Z helped K.W to perform binding assays and immunoprecipitation. K.W, I.B, wrote the manuscript with help of A.C, AR.B, MG.G, R.B, assisted in production, purification and characterization of RSV M2-2 protein. T.B. generated OGG1 knock out hSAECs using CRISPR/Cas9 genome editing. R.G., A.C provided purified characterized suspensions of RSV virions and advised RSV infection of animals. A.B, R.Z. and S.V designed, performed, and analyzed protein-protein interaction assays. AR.B and X.B advised RNA CLIP, and RNA-IP experiments. All authors discussed results and approved the content of the manuscript.

Corresponding author: Istvan Boldogh, DM\&B, PhD Department of Microbiology and Immunology School of Medicine, University of Texas Medical Branch at Galveston, Texas 301 University Blvd, Galveston, Texas 77555, USA Email: sboldogh@utmb.edu 
38 Permanent addresses

39 a Key Laboratory of Molecular Epigenetics of Ministry of Education, School of Life Science,

$40 \quad$ Northeast Normal University, Changchun, Jilin 130024, China

41 bBiomedical Pioneering Innovation Center, School of Life Sciences, Peking University, Beijing

42 100871, China

43 'Department of Immunology, Faculty of Medicine, University of Debrecen, Debrecen, Hungary,

$44 \quad \mathrm{H}-4032$

$45 \quad{ }^{\mathrm{d}}$ Research Institute of Sport Science, Semmelweis University, Budapest, Hungary

46 eHoremeio Research Laboratory, at First Department of Pediatrics, National and

47 Kapodistrian, University of Athens, Greece

48

49

\section{Conflict of interest}

The authors declare that no conflict of interest exists

51

52 
Respiratory syncytial virus (RSV) infection induces an oxidizing environment linked to increased viral load, expression of pro-inflammatory genes, and excessive lung inflammation.

57 The mechanisms of how reactive oxygen species (ROS) promotes viral gene expression have 58 remained largely elusive. Here we show that nascent (n)RNAs of RSV acquire 8-oxo-7,8-

59 dihydroguanine (8-oxo(r)Gua) -a covalently modified guanine base in their 5'-UTR

60 peritranscriptionally, while paired with the 3'-terminus of viral gene(s). 8-oxo(r)Gua is bound by

61 8-oxoguanine DNA glycosylase1 (OGG1), a complex that physically interacts with and recruits

62 the anti-terminator protein M2-1 to increase viral gene transcription. Knockdown of OGG1 (but

63 not other DNA glycosylases) or inhibition of its binding, significantly decreased RSV mRNA,

64 protein levels and yield of progeny in cultured cells and airways. Collectively, these data

65 suggest that Gua oxidation in vRNA, serves as an epitranscriptomic mark that repurposes

66 OGG1 to increase lytic viral replication. Pharmacological inhibition of OGG1 binding to the

67 epitranscriptomic mark could have clinical utility to decrease manifestations of RSV infection. 68 


\section{Introduction}

Respiratory syncytial virus (RSV) is one of the most frequent causes of severe lower respiratory tract infections ${ }^{1}$. Worldwide, RSV infection is responsible for upper/lower clinical manifestations in nearly all children under age two, resulting in over 3 million hospitalizations and nearly 200,000 in-hospital deaths ${ }^{2}$. In the U.S., more than 100,000 children are hospitalized yearly due to severe consequences of RSV infection, making it the most frequent disease among hospitalized infants ${ }^{3}$. Re-infections with RSV may occur throughout life and are mostly limited to the upper airways. However, in immunocompromised adults and the elderly, RSV infection spreads into the lower airways, producing severe bronchiolitis and pneumonia, respiratory morbidity and mortality ${ }^{4,5}$. RSV infections produce clinical impact similar to that seen with influenza, rhino-, and coronaviruses ${ }^{2,6}$. No effective vaccine or drug is currently available, and patient's treatment is limited to supportive medical care ${ }^{7}$.

RSV infects airway epithelial cells by binding cellular receptors, producing envelope fusion with the plasma membrane, delivering nucleocapsids into the cytoplasm ${ }^{8,9}$. Once internalized, the negative-sense viral genome encodes for ten mRNAs regulated by 3' and 5' noncoding sequences, transcribed by the RNA-dependent RNA polymerase complex [RdRp; large polymerase $(L)$, phosphoprotein $(P)]$, that are translated into eleven proteins ${ }^{10}$. $R d R p$ initiates transcription and replication of nascent (n)RNA transcripts using the same genomic template including vRNA capping (via the conserved region $V$ of the $L$ protein), and polyadenylation ${ }^{10-12}$. RdRp recognizes the gene start (GS) and end (GE) of each gene on the genome, guiding mRNA synthesis ${ }^{13,14}$, which is modulated by the anti-terminator matrix protein 2 (M2-1) also called the transcription elongation factor ${ }^{15}$. The primary cellular sites of RSV transcription and replication are cytoplasmic inclusion bodies (IBs) ${ }^{16}$.

Efficacy of vRNA transcription and replication are also dependent on cellular factors ${ }^{17}$. For example, host cytoskeletal, membrane, heat-shock proteins or those involved in protein trafficking have all been associated with RSV replication ${ }^{18-20}$. Additionally, cellular enzymes, including methyl- and acetyl-transferases, deposit/induce covalent base modifications to vRNAs

97 (epitranscriptomic marks; e.g., N6-methyladenosine (m6A), 5-methylcytidine (m5C), or N4-

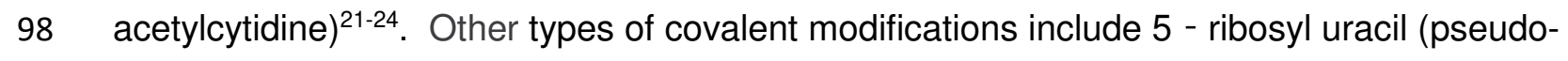
uridine) and inosine also generated enzymatically; however, there are no "reader" or "eraser" enzymes identified to date ${ }^{24}$. Epitranscriptomic marks facilitate various steps in viral replication, viral mRNA stability, translation, and/or shield viral RNAs from recognition by host RNA-specific innate receptors ${ }^{21,22,25}$. Similar epitranscriptomic modifications control viral latency and lytic replication of DNA viruses ${ }^{26}$. 
RNAs are 10-25-times more susceptible to oxidation than DNA in vivo due to their single-stranded nature, their lack of or partial association with protecting proteins, and their close proximity to sites of ROS generators ${ }^{27-29}$. In an oxidizing cellular milieu, ROS primarily attack guanine (Gua) heterocycles due to their having the lowest oxidation potential among nucleic acid bases ${ }^{30}$. Gua oxidation results in 8-oxo-7,8-dihydroriboguanine (8-oxo(r)Gua) or 8oxo-7,8-dihydrodesoxyguanine (8-oxo(d)Gua) in RNA and DNA respectively ${ }^{31}$. The mutagenic 8-oxo(d)Gua lesions are removed from DNA by 8-oxoguanine DNA glycosylase ${ }^{32}$, however, in RNA it may affect codon-anticodon pairing during translation, or when in excess, induce apoptosis through cellular RNA surveillance mechanisms ${ }^{28,33}$. In vRNA, oxidation at Gua is unexplored; however, the addition of antioxidants or inhibition of ROS production significantly lowers RSV as well as human metapneumo-virus (HMPV) replication ${ }^{34-37}$, raising the potential for 8-oxo(r)Gua playing roles in vRNAs.

The aim of the present study was to examine the role(s) of oxidative base modification(s) particularly to Gua in the RSV transcriptome and understand its consequences. Our results show that RSV infection-induced ROS co-transcriptionally generated 8-oxo(r)Gua in the 5'UTRs of nascent vRNAs while paired with the 3'-end of genes. 8-oxoguanine DNA glycosylase1 (OGG1), an oxidized Gua base specific DNA repair protein ${ }^{32,38}$, physically interacted with 8-oxo(r)Gua in vRNAs and recruited the RSV-encoded transcription elongation factor M2-1 to promote mRNA synthesis. Knockdown of OGG1 (but not other repair proteins) or inhibition of OGG1 binding to the oxidatively modified Gua(s) decreased viral mRNA, protein levels as well as yield of RSV progeny (100 to 1000-fold) in both airways and cultured cells. These data imply that RSV has adopted cellular biosynthetic machinery via Gua oxidation in VRNA as an epitranscriptomic marker to recruit the cellular reader, OGG1 for efficient virus

127 replication. Our findings not only provide a new mechanistic understanding the effects of ROS 128 on vRNA transcription, but point to the potential utility of OGG1 inhibitors in drug development 129 for clinical purposes. 


\section{Oxidatively modified guanine base lesions in viral mRNAs}

Given the profound effect of oxidative stress on RSV replication, pathogenesis, ${ }_{1}$ as well as vulnerability of Gua(s) to $\operatorname{ROS}^{28,36}$, we examined oxidative modification to vRNA by assessing Gua base modification(s). The immortalized human small airway epithelial cell cultures (hSAEC -a cell type target of RSV infection involved in disease pathogenesis ${ }^{39,40}$ ) were infected with RSV and harvested to isolate RNAs using buffers containing the iron chelator desferioxamine (DFO) to prevent RNA oxidation ${ }^{41}$. Purified RNAs were incubated with 8oxo(r)Gua-specific antibody $(\mathrm{Ab})^{41}$, cross-linked and samples were immunoprecipitated (IP-ed, RNA-IP). In parallel, (FLAG)OGG1-expressing hSAECs were infected as above and vRNA associated with proteins was cross-linked and chromatin-IP-ed (CLIP-ed) with Ab to the FLAG epitope tag. RNA-protein crosslinking was performed using formalin, to avoid ultraviolet (UV) irradiation-induced generation of ROS and oxidative modifications to the RNA ${ }^{42}$. In controls, transcriptional processivity and anti-termination factor (M2-1) and nucleoprotein $(\mathrm{N})$ proteins associated vRNAs were CLIP-ed using the corresponding Abs. IP-ed and CLIP-ed RNAs were isolated and converted into cDNA using oligo-dT primers to assess abundance of transcripts for nonstructural protein-1(NS1), N, attachment glycoprotein $G(G)$, fusion protein (F) and L using quantitative real time (qRT)-PCR (primer sequences: Table 1; primer validation and amount of RNA used in qRT-PCR were determined in preliminary studies (Supplementary Fig. 1a,b). Results show that Abs to 8-oxo(r)Gua or FLAG(OGG1) enriched all RSV mRNAs tested (Fig. 1a). The level of IP-ed mRNA was the highest for NS1 (encoded by the 3'-end proximal gene) (Fig. 1a, most left panel), and mRNA levels from subsequent genes were decreased, -the most promoter-distal L gene product was the least (Fig. 1a, most right panel). These data are in line with those showing that RdRp re-engages with less efficiency the more distant genes from the 3'-end of the genome ${ }^{14,43}$. In controls, Ab to M2-1 IP-ed viral mRNAs, similar to that of CLIP-ed, IP-ed by $A b$ to FLAG(OGG1) and Ab to 8-oxoGua, while levels of N protein-Ab IP-ed viral mRNAs were similar to that of IgG (Fig. 1a). These results are in agreement with M2-1' RNA binding core domain and its roles in de novo mRNA synthesis ${ }^{44}$ and those showing that the $\mathrm{N}$ protein binds genome and anti-genome as they have encapsidation signals, which are absent in $\mathrm{mRNAs}^{10}$. There was no interaction observed between viral mRNA the repair protein MTH1 (a human homolog of $E$. coli Mutator 1), an enzyme that specifically recognizes 8-oxo(r)Gua generated in the cytoplasmic ribonucleotide pool ${ }^{45}$.

To obtain information on the cellular site of vRNA oxidation, cells were infected as above, and dual-color microscopic imaging was performed $24 \mathrm{~h}$ post-infection. IBs were 
visualized using $\mathrm{Ab}$ to $\mathrm{N}$ protein (green, Alexa Fluor 488) and then co-stained with $\mathrm{Ab}$ to 8oxo(r)Gua (red, Alexa Fluor 594). These data show that oxidatively modified RNAs at Gua(s) primarily co-localized to IBs, although they are also found in the cytoplasm (Fig. 1b). In support of specificity, our analysis showed that oxidatively modified proteins (carbonylated and nitrosylated) were seen at the periphery and/or adjacent to IBs (Fig 1C,d). Because IBs are the sites of vRNA synthesis, these results also imply that oxidative modification to Gua in the RSV transcriptome possibly occurs co-transcriptionally, either by direct oxidation or by incorporation of oxidatively modified Gua nucleoside triphosphate (8-oxo(r)GuaNTP). The latter may be due to the lack of an innate capacity of RdRp to differentiate between GNTP and 8-oxo(r)GuaNTP, similar to mammalian RNA polymerases ${ }^{46}$.

\section{OGG1 converts oxidatively modified Gua into enhanced RNA expression}

Based on binding of 8-oxo(r)Gua-Ab to and association of OGG1 with vRNAs (Fig. 1a, b, c), we investigated whether the oxidative Gua modification plays a role in RSV RNA transcription. To test our hypothesis, 8-oxo(r)Gua in vRNA was decreased by normalizing RSVinduced ROS through treatment with AOs, EUK-8 (100 or $500 \mu \mathrm{M})$ or N-acetyl-L-cysteine [NAC, a GSH precursor) at 10 or $20 \mu \mathrm{M})]^{34}$. Total RNAs were isolated, IP-ed using Ab to 8-oxoGua and converted into cDNA for qRT-PCR. Results showed that AO treatment significantly lowered ROS levels (Supplementary Fig. 2d), and abundance of 8-oxo(r)Gua-containing mRNA coding for the attachment protein G, compared to that isolated from infected mock-treated cells (Fig. 2a). Similarly, there were lower mRNA levels of NS1, N, G, F and L (Fig. 2b) and genomic vRNA (Fig. 2c), as well as viral progeny (Fig. 2d). This phenomenon was not restricted to hSAECs, as we found that a decrease in 8-0xo(r)Gua levels correlated well with RSV replication (mRNA, genome and progeny) in established airway epithelial cells, A549, which is a model of infection (Supplementary Fig. 2a,b,c). In controls, ribavirin, a guanosine analog, an RdRp inhibitor producing co-transcriptional inhibition ${ }^{47}$ was used, which showed an inhibitory effect irrespective of cell type (Fig. 2b,c,d; Supplementary Fig. 2a,b,c).

Results showing that decrease in ROS levels led to a lower abundance of 8-oxo(r)Guacontaining vRNA and physical association of OGG1 with vRNAs (Fig. 1a,c) suggested to us that oxidatively modified Gua along with OGG1 has a role in viral replication. To test OGG1 was siRNA-silenced or permanently knocked out by CRISPR-Cas9 genomic editing in hSAECs

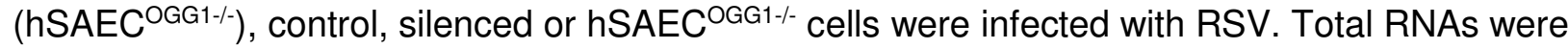
isolated, converted into cDNA, and vRNA levels were assessed by qRT-PCR. Results showed significantly lower mRNA (NS1, N, G, F and L) levels in OGG1 silenced hSAECs (Fig. 2e) and 
hSAEC ${ }^{\text {OGG1-/ }}$ cells (Fig. 2f). Additionally, there were decreased levels of M2-1 and N proteins in knockout cells compared to those expressing OGG1 as determined by Western immunoblotting (Wb) (Fig. 2g, left and right panels). Similar results obtained using OGG1-silenced A549 cells suggested that the observed effects are not restricted to hSAECs (Supplementary. Fig. 3a). Absence of OGG1 significantly decreased levels of genomic RNAs in hSAECs and A549 cells at 12h, 24h and 36h post-infection (RNA levels at $2 \mathrm{~h}$ post-exposure served as input, Fig. $\mathbf{2 h}, \mathbf{i}$ and Supplementary Fig. 3d). Lack of OGG1 led to a more than 10-fold decrease in viral yield in hSAEC (Fig. 2j, k) and A549 cells (Supplementary Fig. 3b). Transgenic expression of OGG1 in hSAEC ${ }^{\text {OGG1-/ }}$ restored RSV output to a level similar to that of OGG1 expressing cells (Fig. 2k). Silencing other DNA glycosylases, endonuclease VIII-like 1 (NEIL1, recognizes and repairs oxidatively modified ring-fragmented purines, pyrimidines, 5-hydroxyuracil $\left.{ }^{48}\right)$, or the 8oxo(r)Gua-specific MTH1 ${ }^{45}$ had no effect on RSV replication (Supplementary Fig. 3a). These results suggest that OGG1 bound to 8-oxo(r)Gua in RNA is actively utilized to maximize output of RSV infection.

To examine whether changes in cellular physiology due to OGG1 depletion (selection, puromycin) affected RSV replication, we inhibited OGG1 binding to 8-oxo(r)Gua in vRNAs by using the selective binding-site inhibitor, TH548749. hSAECs were RSV-infected and treated with TH5487 (10 $\mu \mathrm{M}$ or the equivalent volume of solvent). RNAs isolated were subjected to qRT-PCR analysis. Results show that TH5487 treatment of infected cells significantly decreased mRNA levels coded by NS1, N, G, F, and L genes (Fig. 3a). Consistent with these data, we observed significant decreases in the levels of $\mathrm{N}$ and M2-1 proteins (Fig. 3b, left and right panels) and genomic RNA ( 24 and 36 hpi; Fig. 3c) levels. The Inhibitory effects of TH5487 were not cell type specific because it also inhibited RSV output in A549 cells (Supplementary Fig. 3b,c,f,h). Inhibition of OGG1's binding to oxidatively modified Gua in vRNA decreased RSV-yield in a concentration dependent (5, $10 \mu \mathrm{M}$ ) manner in hSAECs (Fig. 3d) and in A549 cells (Supplementary Fig. 3b). TH2840, an inactive analog of TH5487 or O8, which inhibits only OGG1's enzymatic activity ${ }^{49,50}$, had no effect on RSV titers (Fig. 3d, Supplementary Fig. 3b). In line with this, TH5487 inhibited RSV-induced cytopathology while TH2840 and O8 had no effect (Supplementary Fig. 3c). Treatment with TH5487, TH2840 or O8 showed no toxicity in hSAECs or A549 cells (Supplementary Fig. 3i, left and right panels).

To validate the data derived from cultured cells, mice were challenged with purified RSV (106 PFU/lung) and mock treated or treated with TH5487 at $-2 \mathrm{~h},+1 \mathrm{~h}$ and $12 \mathrm{~h}$ intervals over $96 \mathrm{~h}$ (30 mg/kg, intraperitoneal (i.p) ${ }^{49}$. At $2 \mathrm{~h}, 48 \mathrm{~h}$ and $96 \mathrm{~h}$ post-challenge, the lungs were harvested and total RNAs isolated. The RNAs were converted into cDNA by using oligo-dT and random 
primers to assess RSV mRNA and genomic RNA levels, respectively. Quantitative RT-PCR results showed that the inhibition of OGG1's substrate binding significantly lowered levels of $N$, G, and F mRNAs, and genomic RNA compared to mock-treated RSV-infected animals (Fig. 3f, g). There was no sign of TH5487 toxicity in infected animals as shown previously ${ }^{49}$. Data from cell culture and animal experiments strongly suggest that OGG1 binding to oxidatively modified Gua in viral RNAs is essential for efficient RSV replication. Future studies will determine the clinical potential of small molecule inhibitor(s) of OGG1.

\section{The 5'-end of nRNAs are hotspots of oxidative Gua modifications and the preferential site of OGG1 binding}

To determine the site(s) of oxidative modification of Gua in vRNAs, hybridization-coupled electro-mobility shift assays (EMSA) were performed. We designed synthetic Cy5-labeled $40 \mathrm{nt}$ long DNA probes homologous to the 3'-end (from 4552 to $4592 \mathrm{nt}$; containing GS) and the 5'end (from 5433 to 5474 nt, containing GE) of the G genomic RNA (sequences are complementary to the G mRNA) (Fig. 4a, Table 3). G:C contents of these probes were $45 \%$ and $47 \%$, respectively. The sequence of the third probe is homologous to the region located between GE of small hydrophobic protein (SH) and GS of G gene (4506-4546 nt; intergenic sequences complementary to antigenome). The RNA isolated from RSV-infected cells was incubated with individual probes, denatured at $95^{\circ} \mathrm{C}$ for $5 \mathrm{~min}$, and hybridized. Un-hybridized probes and RNAs were digested with mung bean nuclease ${ }^{48}$, then OGG1 was added, crosslinked and mixtures were subjected to EMSA. Results showed that OGG1 extensively bound to the probe-RNA hybrid formed at the 5'-end of viral mRNA, while a weak hybridization signal was observed at the 3'-end of mRNAs (Fig. $\mathbf{4 b}$, lane 6). The probe that was complementary to antigenome gave a weak signal, suggesting that the hybridization observed at the 3'-end of mRNA may be considered as background signal (Fig. 4b, lane 6). RNase-H completely eliminated EMSA signals (Fig. 4b, lane 7, 8 and 9), indicating that OGG1 specifically bound to the 5'-end of the mRNA region that acquired the oxidative modification of Gua, 8-oxo(r)Gua. Accordingly, OGG1 bound to the synthetically made DNA-RNA hybrid containing 8-oxo(r)Gua in the RNA strand in a concentration dependent manner (Fig. 4c). The active-site inhibitor, TH5487, prevented OGG1 binding to the DNA-RNA hybrid containing 8-oxo(r)Gua, lending additional support for binding specificity (Fig. 4d). These results suggest that oxidative modification of Gua occurs at the 5'-UTR of mRNAs.

Binding of OGG1 to the DNA-RNA hybrid at the 5'-end of mRNA suggests that oxidative modifications of Gua occur in the RNA complementary to GS in the genome. To test this 
possibility, we designed Cy5-tagged single stranded (ss) 40 nt RNA probes with and without 8oxo(r)Gua complementary to the 5'-end of the G protein gene. Based on the documented nature of charge migration ${ }^{51}$, the probes contained 8-oxo(r)Gua at the 3'-end of G stretches (5'GGGG*CAAAU-3') of the 5'-UTR. To mimic a dsRNA structure formed during vRNA synthesis ${ }^{10,52}$, the sequence of the ssRNA probes is identical to the sequence of nRNA, which allows it to anneal with the complementary sequence GS of genomic G gene (4552 to $4592 \mathrm{nt}$ ). Results from EMSAs show that OGG1 binds 8-oxo(r)Gua containing dsRNA and not ssRNA (Fig. 4e, lanes 6,7,8), an interaction that could be prevented by TH5487 (Fig. 4f,g). OGG1 had no enzymatic activity on dsRNA (Fig. 4h, lanes 2,3,4), while it efficiently excised damaged bases from dsDNA ${ }^{32}$ (Fig. 4h, lanes 6,7,8; Supplementary Fig. 4a). Therefore, removal by OGG1 of 8-oxo(r)Gua (cleavage of the N-glycosylic bond in RNA) and subsequent degradation of RNA is an unlikely scenario in cellulo. These results also imply that OGG1 interacts with 8oxo(r)Gua formed co-transcriptionally at the transcription active site.

\section{OGG1 physically interacts with the transcriptional elongation factor M2-1}

In eukaryotic cells OGG1 binds to 8-oxo(d)Gua, an epigenetic-like mark in gene promoters, and physically interacts with transcription factors, including NF-kB, to facilitate its DNA occupancy ${ }^{53}$. By analogy, we propose that OGG1 binds to 8-oxo(r)Gua at the 5'-end of RNA and interacts with viral protein(s), potentially aiding efficiency of transcriptional machinery. To test this possibility, FLAG-tagged OGG1, (FLAG)OGG1, expressing hSAECs were infected with RSV, lysed and IP-ed using Ab to FLAG. Complexes that were IP-ed were subjected to $\mathrm{Wb}$ analysis using a polyclonal Ab to RSV proteins. The results showed that (FLAG)OGG1 coIP-ed with M2-1 ( 25 kDa subunit of the tetrameric M2-1 is shown after SDS-PAGE; (Fig. 5a). To confirm this, M2-1 was identified using a monoclonal Ab to M2-1 (Fig. 5b). The lower panel of Fig. 5a and 5b shows intrinsic and transgenic expression of OGG1 using Ab to OGG1. We also noted that there was another $\sim 40$ kDa OGG1-interacting protein immuno-precipitate (Fig. 5a, "right panel); however, its identity remains unknown. To test whether OGG1 directly interacts with M2-1, we purified it to homogeneity ${ }^{54}$ (Supplementary Fig. 4b,c), mixed it with OGG1, and performed pull-down assays. Results showed that OGG1 pulled down M2-1 protein, and Ab to M2-1 IP-ed with OGG1 (Fig. 5c,d), suggesting a direct interaction between M2-1 and OGG1. There was no interaction between M2-1 and MTH1, which was used as control protein (Fig. 5e). OGG1 typically localizes to the nucleus and mitochondria ${ }^{32}$, while M2-1 is found in the cytoplasm, primarily in virus-induced IBs ${ }^{15,55}$. To obtain information on the site of the OGG1-M21 interaction in cellulo, microscopic imaging (immunohistochemistry; $\mathrm{IHC}$ ) and in situ proximity 
ligation assays ${ }^{56}$ were performed using RSV infected hSAECs. Time course studies showed an accumulation of OGG1 in the cytoplasm of RSV-infected cells from $6 \mathrm{hpi}$, as shown by IHC and $\mathrm{Wb}$ analysis (Fig. 5f, g, Supplementary Fig. 4d), while its level did not change in the nuclear compartment (Fig. 5g, Supplementary Fig. 4e, upper panels), suggesting that de novo synthesized OGG1 remained in the cytoplasm. After overlaying IHC images, strong colocalization of OGG1 and M2-1 was observed, especially in IBs. This observation was confirmed by calculating the Pearson fluorophore-moment correlation coefficient ${ }^{57}$. E.g., in contrast to 6 hpi, correlation coefficient $(R)$ at 12,24 and 42 hpi were $R_{-}=0.93, R=0.93$ and $R=0.89$, respectively (Fig. 5f, most right-hand panels). The positive proximity ligation assays strongly suggest there was a physical interaction between OGG1 and M2-1 within IBs (Fig. 5h,i).

\section{OGG1 recruits M2-1 to the transcriptionally active site(s) at the 5'-end of VRNA}

The physical interaction observed between OGG1 and M2-1 in vitro and in cellulo (Fig. $\mathbf{5 a , b , c , d , f )}$ and the fact that OGG1 bound to 8-oxo(r)Gua at the 5'-end of nRNA (Fig. $\mathbf{4 b}, \mathbf{c}, \mathbf{d}$ ), suggest that OGG1 may promote interaction of M2-1 with the transcriptionally active site at GS.

To model this scenario, we utilized ss and ds RNA probes containing \pm 8 -oxo(r)Gua. The presence or absence of 8-oxo(r)Gua in ss or ds RNAs had no effect on M2-1 binding (Fig. 6a). M2-1 bound to ssRNA and dsRNA as a tetramer (arrow heads) and also formed higher molecular size complexes (Fig. 6a) similar to those described for glutaraldehyde cross-linked forms of RNA-M2-1 complexes $^{58}$. Compared to ssRNA (Fig. 6a, lane 1,2) there was a 25 -fold decrease in M2-1's association with dsRNA \pm 8 -oxo(r)Gua (Fig. 6a, lane 3, 4). To test whether M2-1 binding to dsRNA was specific, we show that Ab to M2-1 super-shifted all M2-1-dsRNA complexes (Fig. 6b, lane 3). Ab to OGG1 inhibited its binding to 8-oxo(r)Gua containing dsRNA Fig. 6b, lane 2). IgG was used as control (Fig. 6b, lane 1).

To examine if OGG1 promotes binding of M2-1 to RNA, OGG1 was pre-incubated with a dsRNA probe \pm 8 -oxo(r)Gua and M2-1 was added. OGG1 increased M2-1 occupancy on 8oxo(r)Gua-containing dsRNA in a concentration dependent manner (Fig. 6c, lanes 6 to 10 and Fig. 6c, right panel), while without 8-oxo(r)Gua in the dsRNA, OGG1 had no effect (Fig. 6c, lanes 1 to 5 and Fig. 6c). Time course experiments showed that OGG1 accelerated the interactions between 8-oxo(r)Gua-containing dsRNA and M2-1 (Fig. 6d, lanes 7 to 11 and Fig. $6 \mathbf{d}$ right panel). For instance, the level of dsRNA-associated with M2-1 was similar at $t=1 \mathrm{~min}$ in the presence of OGG1 to that of $\mathrm{t}=6$ min without OGG1 (Fig. 6d, lanes 2 and 7 , Supplementary Fig $\mathbf{4 g}$ ). Interestingly, there was no M2-1-OGG1-dsRNA complex observed; however, OGG1 formed a separate complex with dsRNA (Fig. 6d, arrow). Active site inhibitor 
(TH5487) decreased the effect of OGG1 on the interaction between M2-1 and dsRNA in a concentration dependent manner (Fig. 6e, right panel). Without 8-oxo(r)Gua OGG1 had no effect (Fig. 6c, lanes 1 to 5 and right panel), and in the absence of OGG1, TH5487 had no effect on M2-1 binding (Fig. 6f). These data imply that prior OGG1 binding to dsRNA is needed for efficient association of M2-1 with dsRNA. In control experiments, MTH1 had no effect on M21 binding to dsRNA (8-oxo(r)Gua:C) (Supplementary Fig. 4h). From these results, we speculate that OGG1 binds to dsRNA - a hybrid between nRNA and the 3'-end of viral gene(s) via co-transcriptionally generated 8-oxo(r)Gua, where it interacts with and recruits $\mathrm{M} 2-1$ to the transcriptionally active site(s). OGG1 had no effect on the interaction of M2-1 with ssRNA containing 8-oxo(r)Gua (Supplementary Fig. 4f).

We next explored the fate of OGG1 once the full-length mRNA disengages from genome. To do so, mRNAs isolated form RSV-infected cells were mixed with His-tagged OGG1 in binding buffer, and IP assays were performed. Levels of mRNA encoding for G protein in IPs was determined using qRT-PCR. Compared to IgG, Ab to His-(OGG1) had enriched viral RNAs coding for $\mathrm{G}$ protein in IPs (Supplementary Fig. 4j). Heat denaturation of RNA $\left(65^{\circ} \mathrm{C}\right.$ for $5 \mathrm{~min}$ ) prevented OGG1 binding. In controls, M2-1 extensively IP-ed with G-mRNA, while N protein lacked interaction with mRNA (Supplementary Fig. 3j). Taking that the 5'-end of nRNAs is the preferential site of OGG1 binding (Figs $\mathbf{4 b , c , d}$ ), we performed a series of experiments that examined OGG1 binding to a Cy5-tagged $40 \mathrm{nt}$ base-long ssRNA (identical to 5-end of GmRNA). Results showed that OGG1 bound to the non-denatured RNA probe only when it contained 8-oxo(r)Gua (Fig. $\mathbf{6 g}$, lanes: 5 to 8) similar to dsRNA (Fig. $\mathbf{6 g}$, lanes: 9 to 12). Heat denaturation $\left(65^{\circ} \mathrm{C}\right.$ for $5 \mathrm{~min}$ ) of RNA abolished OGG1 binding (Fig. $\mathbf{6 g}$, lanes 1 to 4 ) implying that OGG1 binds to the substrate via temperature-sensitive secondary RNA structure(s) at the 5-end of G-mRNA. The predicted secondary structure of the probe is shown in Fig. $\mathbf{6 g}$ (right panel). A secondary structure of G-mRNA is shown in Supplementary, Fig. 4i as predicted by "RNAstructure" software ${ }^{59}$. OGG1 lacks enzymatic activity on dsRNAs (Fig. 4h, Supplementary Fig. 4a), therefore we speculate that OGG1 remains in complex with full-length viral mRNAs (Fig. 6h). The post-transcriptional roles of OGG1 is a matter for future studies. 


\section{Discussion}

RSV is one of the leading causes of life-threatening respiratory system illnesses globally in infants, preschool children, immunocompromised adults and the elderly; yet, treatment is mostly limited to supportive medical care ${ }^{1,4,7}$. RSV infection-induced generation of ROS is linked to expression of inflammatory genes and severity of clinical disease; however, the mechanism by which the oxidizing environment modulates virus replication remains unknown. Here we report that vRNAs acquire ROS-generated 8-oxo(r)Gua as an epitranscriptomic mark that is "read" by the repurposed DNA repair protein OGG1. Inhibition of 8-oxo(r)Gua generation through AOs, OGG1 knockdown or inhibition of its binding to epitranscriptomic mark significantly decreased the level of viral mRNAs, proteins, and viral progeny in cultured cells and airways. Reconstitution experiments show that OGG1 binds to the 5'-UTR of nRNAs containing 8oxo(r)Gua while paired with the 3'-end of viral gene(s) interacts with and recruits the transcriptional elongation factor, M2-1 to transcriptionally active sites. Taken together, these data suggest that ROS generated in RSV-infected cells is utilized to oxidize Gua in vRNA, which serves as an epitranscriptomic mark that repurposes OGG1 to enhance RSV replication. These studies also illustrate how the virus usurps cellular proteins to enhance its replication.

RNAs are highly vulnerable to oxidative modifications, due to their single stranded nature, partial protection by RNA-binding proteins, and unprotected Watson-Crick edges of nucleotides $^{27-29}$. The nucleobase of guanosine is especially vulnerable to modifications as it has the lowest oxidation potential ${ }^{63}$. Therefore, 8-oxoGua is often generated in RNAs and in nucleotide triphosphate pool by $\mathrm{ROS}^{28}$, in an oxidizing intra-cellular environment, such as in RSV-infected cells ${ }^{36}$. Therefore, association of lesion-specific OGG1 with or IP by 8-oxoGua Ab of mRNAs of NS1, N, G, F and L genes in RSV-infected cells is not surprising. Importantly, the level of OGG1-associated vRNA was similar to that of RNA that was IP-ed using Ab to M2-1. M2-1 interacts with RNA via its zinc-binding and core domains ${ }^{44,60}$. OGG1 lacks a zinc-finger motif ${ }^{61}$, so its interaction with vRNA likely occurs through its recognition domain, which is specific for 8-oxoGua in DNA and RNA.

Although oxidative modification to Gua can occur at any Gua islet (GGG, GGGG) within genomic RNA and mRNAs, hybridization-coupled EMSA clearly indicated that 8-oxo(r)Gua was formed at the 5'-end of viral nRNAs. There were only insignificant levels of 8-oxo( $r$ )Gua in genomic RNA. These findings are in line with the sequence 5'-GGGGCAAAU-3' at the 5'-UTR of NS1, NS2, N, P, M, SH, G, F, and M2, and sequence 5'GGGACAAAAU-3' of L mRNA ${ }^{10,13}$. The exact position of 8-oxo(r)Gua in these islets is yet to be determined; however, based on the previously characterized nature of charge migration, it is most likely located at the end of $\mathrm{G}$ 
stretches ${ }^{51}$. It is known that during viral replication, the dsRNA hybrid may reach 30 nucleotides or more. For instance, in the case of VSV, mRNA is separated from the genome and then capped after reaching 31 nucleotides ${ }^{52}$. Thus, to reconstitute an in cellulo scenario, 8-oxo(r)Gua was synthetically placed at the end of 3'-end of G series in a 40-nucleotide long RNA that is complementary to the 3'-end of genomic RNA (probe), which mimics dsRNA (probe) similar to the polymerization active site at GS ${ }^{52}$. Binding of OGG1 to dsRNA with 8-oxo(r)Gua was similar to DNA containing 8-oxo(d)Gua. Although M2-1 is an RNA binding protein, it showed poor interaction with dsRNA \pm 8 -oxo(r)Gua compared to ssRNA \pm 8 -oxo(r)Gua. However, OGG1 significantly increased M2-1 binding (over 10-fold) only to dsRNA containing 8-oxo(r)Gua. The exact molecular mechanism by which OGG1 enhances M2-1 binding is yet to be elucidated; however, it is possible that OGG1-induces structural changes in dsRNA that are creating a specific binding interface in the hybrid between the genome and nRNA that facilitates M2-1 interaction with the polymerization complex (Fig. 6h). This scenario could be similar to that seen in mammalian cells, where OGG1 binds to the epigenetic mark 8-oxo(d)Gua in gene regulatory regions, thereby serving as a nucleation site and increasing DNA occupancy of transcription factors ${ }^{64,65}$. During mRNA synthesis, M2-1 interacts with $P$ protein and vRNA in a competitive "on and off" manner, because the RNA recognition epitopes of M2-1 overlap with the site interacting with $P$ protein ${ }^{66}$. Together with the data showing that OGG1 increases RSV transcription, it is conceivable that OGG1 not only interacts physically with M2-1 but also facilitates its stable interaction with vRNA. Indeed, in OGG1 proficient cells, the level of mRNA was approximately 10-times higher, compared to cells lacking OGG1 or treated with the small molecule inhibitor of OGG1. These data also imply that the lack of OGG1 or the OGG1 inhibitor prevents efficient assembly of transcriptional initiation complex and transcriptional elongation in cultured cells and in lungs.

Based on these data, 8-oxo(r)Gua is functionally similar to covalently modified nucleotides, such as $\mathrm{m}^{6} \mathrm{~A}$, and $\mathrm{m}^{5} \mathrm{C}$, in genomic and mRNAs of RNA viruses (e.g., hMPV, RSV, measles, influenza, flavi-, and corona viruses) with fundamental implications in their life cycles ${ }^{21-}$ 23,67,68. In contrast to epitranscriptomic moieties that are deposited and removed by cellular enzymes called "writers" and "erasers", respectively²2, 8-oxo(r)Gua is directly generated by the interaction of Gua with one of several reactive species (primarily hydroxyl radical ${ }^{63}$ ). Thus ROS is considered to be a covalent modifier ("writer") of Gua by oxidizing --subtracting an electron generating the epitranscriptomic mark 8-oxo(r)Gua of which "reader" is the repurposed base specific DNA repair protein OGG1. As a "reader" OGG1 facilitates M2-1 binding to 5'-UTR, thereby promoting mRNA synthesis. Of note, OGG1 has an innate ability to migrate/diffuse 
431 along nucleic acid strands without energy requirement ${ }^{38}$. Thus, OGG1 may migrate along with

432 M2-1 on vRNA to facilitate transcription elongation. Another possibility is that OGG1 remains

433 engaged with the 8-oxo(r)Gua-containing 5'-UTR of mRNA via temperature sensitive secondary

434 stem-loops. If this is the case OGG1-5'-UTR could have roles in initiation of translation similar to

435 those 5'-UTR-protein complexes of influenza, corona-, and flavi-viruses ${ }^{69}$. The propensity of 8-

436 oxo(r)Gua to pair with adenine may promote formation of specific secondary structures or affect

437 the configuration of the viral RNA which, together with OGG1, allow RSV to exploit the host

438 translation machinery.

439 Microscopic and molecular co-localization studies show that 8-oxo(r)Gua-containing

440 vRNAs primarily localized to IBs, highly dynamic structures in the cytoplasm. Interestingly,

441 carbonylated and nitrosylated proteins are seen at the periphery and/or adjacent to IBs.

442 Although these observations ought to be further investigated, one may propose that repurposed

443 host proteins are protecting viral synthetic machinery and vRNAs from excessive oxidative

444 modifications. The observation that RNAs containing 8-oxo(r)Gua localize within IBs, along with

445 supporting data shown here, implies that oxidative modifications caused by ROS of Gua in the

446 RSV transcriptome occur co-transcriptionally. Another important observation is that OGG1, a

447 nuclear protein, accumulated in the cytoplasm of RSV-infected cells and co-localized with M2-1

448 within IBs, where components of the RNA polymerase complex proteins $(P, L)$ are located.

449 Interestingly, OGG1 level in the nucleus was unchanged suggesting that the newly made OGG1

450 remained in the cytoplasm. Because cytoplasmic and nuclear extracts consistently showed the

$451 \sim 39$ kDa OGG1, we exclude the possibilities that new variants were made by alternative splicing

452 or that the mitochondrial OGG1 $(42.5 \mathrm{kDa})$ is involved. The molecular mechanism by which

453 OGG1 is targeted to IBs remains unclear, but we note that site-specific posttranslational

454 modifications (acetylation, phosphorylation, O-GlcNAcylation and others) of OGG1 could be the

455 culprit. Importantly, the Pearson fluorophore-moment correlation coefficient ${ }^{57}$ clearly showed

456 that OGG1 co-localized with M2-1 within IBs and physically interact as shown by in situ

457 proximity ligation assays and their co-IP from RSV-infected cells.

458 The fate of OGG1 on viral mRNA remains unclear; however, we note that OGG1 has an

459 innate ability to migrate/diffuse along nucleic acid strands without energy requirement ${ }^{38}$. Thus,

460 OGG1 may migrate along with M2-1 on RNA to facilitate transcription elongation. Another

461 possibility is that OGG1 remains engaged with the 8-oxo(r)Gua-containing UTR of mRNA via

462 temperature sensitive secondary stem-loops of RNA (Fig. 6h). For instance, the 5-UTRs of

463 influenza, corona-, and flavi- viruses, involved in initiation of translation, are reported to form 
464 functional protein-RNA complexes ${ }^{69}$. Thus, it is conceivable that OGG1 associated with

465 secondary elements of RSV mRNAs may be used to hijack the cellular translation machinery.

466 This work represents the first study on the importance of an oxidatively modified base

467 lesion in a noncoding region of viral mRNA during the RSV life-cycle. Stretches of Gua in RNA

468 are sink of electrons ${ }^{51}$, and oxidation of Gua may occur at any site(s) of mRNAs and may lead

469 to errors during protein synthesis as reported in mammalian cells ${ }^{70}$. 8-oxo(r)Gua can also be

470 generated in the genome and anti-genome of RSV, although they are encapsidated, a scenario

471 that occurs in chromatin ${ }^{65}$. Previous studies have considered that mutational errors are made by

472 RdRp during replication due to the combination of a lack of proofreading activity and low

473 fidelity ${ }^{71}$. Oxidized Gua in viral genome or antigenome could pair with $A$ and can be responsible

474 for the previously documented variability of RSV strains and their virulence and evolvability ${ }^{72}$,

475 although this needs to be experimentally shown.

476 In conclusion, the data presented here show that 8-oxo(r)Gua in 5'-UTR of vRNAs

477 functions as an epitranscriptomic mark. Its presence in vRNAs is conveyed into function by the

478 repurposed base specific DNA repair protein OGG1. Because OGG1 has no enzymatic activity

479 (erasing activity) on RNAs, OGG1 is considered a "reader" in the context of epitranscriptomic

480 and translates the oxidatively-generated mark into efficient virus replication. Indeed, the

481 absence of either 8-oxo(r)Gua or OGG1 or inhibition of their interaction by small molecule

482 decreased the output of RSV infection. These novel findings not only widen our understanding

483 of the mechanism of RSV gene expression, but also represent new antiviral targets, which may

484 facilitate the development of novel therapeutics. Because of the electron deficient oxidizing

485 environment in virus-infected cells, covalent modifications of Gua could be a common feature in

486 viral RNAs, making these findings potentially applicable to influenza, rhinovirus, corona-, and

487 metapneumo-viruses.

488 
Funding: This work was funded by the NIH National Institute of Allergic and Infectious Diseases NIAID/AI062885 (I.B., AR.B, R.G., A.C); NIH, National Heart, Lung, and Blood Institute R01HL145477 (TK.H., I.B), National Natural Science Foundation of China (grant no. 31970686 to X.B); University of Texas Medical Branch startup funds (to M.G.G.), a Rising Science and

494 Technology Acquisition and Retention Program award (to MG.G.), and the Welch Foundation 495 (H-2032-20200401) (to M.G.G.).

Acknowledgements: Authors are grateful to Dr. Thomas Helleday (Science for Life Laboratory, Department of Oncology-Pathology, Karolinska Institute, S-171 76 Stockholm, Sweden) for providing the OGG1 inhibitor (TH5487) during initial phases of these studies.

We thank Dr. Sherry Haller Editor (Department of Microbiology and Immunology, University of

501 Texas Medical Branch at Galveston) for English editing of the manuscript. 


\section{Material and Methods}

509 Cell cultures. Hep-2 cells (ATCC number CCL-23) were grown in Eagle's minimum essential 510 medium (MEM) containing 10\% (v/v) fetal bovine serum (FBS). A549 cells were grown in

511 Dulbecco's Modified Eagle's Medium/Nutrient Mixture F-12 Ham (DMEM/F12) supplemented 512 with 10\% (v/v) FBS (HYCLONE), $10 \mathrm{mM}$ glutamine, $100 \mathrm{IU} / \mathrm{mL}$ penicillin, and $100 \mathrm{\mu g} / \mathrm{mL}$ 513 streptomycin. Telomerase/CDK4 immortalized human small airway epithelial cells (hSAECs) ${ }^{40}$ were maintained in SAEC growth medium (PromoCell, Item \#:C-21270), containing Growth Medium Supplement Mix (PromoCell Item \#: C-39175), (7.5 mg/mL bovine pituitary extract, 0.5 $\mathrm{mg} / \mathrm{mL}$ hydrocortisone, $0.5 \mu \mathrm{g} / \mathrm{mL}$ human epidermal growth factor, $0.5 \mathrm{mg} / \mathrm{mL}$ epinephrine, 10 $\mathrm{mg} / \mathrm{mL}$ transferrin, $5 \mathrm{mg} / \mathrm{mL}$ insulin, $0.1 \mu \mathrm{g} / \mathrm{mL}$ retinoic acid, $0.5 \mu \mathrm{g} / \mathrm{mL}$ triiodothyronine, 50 $\mathrm{mg} / \mathrm{mL}$ gentamicin, and $50 \mathrm{mg} / \mathrm{mL}$ BSA). Cells were regularly tested for Mycoplasma contamination by the Tissue Culture Core Facility at UTMB (https://www.utmb.edu/microbiology/core-facilities/tccf).

CRISPR/Cas9 genome editing. OGG1 deletion from hSAECs was performed using a Clustered Regulatory Interspaced Short Palindrome Repeat (CRISPR)-associate system (Cas) 9 genome editing as previous described ${ }^{49}$. In brief, targeting sequences of OGG1 5'GATGCGGGCGATGTTGTTGTTGG-3' and 5'-AACAACATCGCCCGCATCACTGG-3' were cloned into pSpCas9 (BB)-2A-Puro expression vector. Following transfection of the vector by Lipofectamine 2000 (Item \#: 11668027, ThermoFisher Sci/Invitrogen) into hSAEC, $3 \mu \mathrm{g} / \mathrm{mL}$ of puromycin (Corning, Item \#: 61-385-RA) was added, cells were sub-cultured into 24-well plates and clones were established. Clones were further examined for OGG1 expression by qRT-PCR and Western immunoblotting. OGG1 knockout cultures were maintained in SAEC growth medium (PromoCell, Item \#: C-21270), containing Growth Medium Supplement Mix (PromoCell, item \#: C-39175) in the presence of $2 \mu \mathrm{g} / \mathrm{mL}$ puromycin (Corning, Item \#: 61-385-RA).

RSV propagation, purification, infection and titration. The human RSV A2 strain (ATCC VR1544) was propagated using HEp-2 cells (ATCC CCL-23). For experiments, cell monolayers

534 (80 to $90 \%$ confluence) were infected with RSV at a pre-calculated multiplicity of infection (MOI). 535 In brief, OGG1-expressing, -depleted or OGG1 knockout cells or those expressing transgenic 536 OGG1 were infected with RSV (strain: A2). Following a $2 \mathrm{~h}$ adsorption period, the unbound virus 537 was removed, the cell monolayer was washed, and culture medium containing $2 \%$ FBS was 538 added. To assess viral gene expression, cells were infected at $\mathrm{MOI}=0.1$ and RNAs were 539 extracted at the times indicated in results. For IPs, and cross-linked coupled IPs cells were 
540 infected at $\mathrm{MOI}=3$. For microscopic imaging ( $\mathrm{IHC}$ and PLA), cells were infected at $\mathrm{MOI}=1$. To

541 generate RSV stock or determine the effect of inhibitors on yields of progeny, cells were

542 infected at $\mathrm{MOI}=0.1$. RSV titration: cells were infected, snap frozen then towed and clarified by

543 centrifugation. Serial (10-fold) dilutions of the supernatant fluids were added to HEp-2 cells

544 grown in 12-well plates (80\% confluence), and viral titers were determined by the

545 methylcellulose plaque assay ${ }^{73}$. To challenge lungs, a purified RSV suspension was used. RSV

546 was purified on discontinuous sucrose gradients (Infant Bronchiolitis and Viral Core. Director Dr

547 Garofalo, Professor, UTMB) as described previously ${ }^{73}$. Aliquots of sucrose purified (cytokine

548 and lipopolysaccharide free) preparations were stored at $-80^{\circ} \mathrm{C}$ until use.

549 Animal challenge and treatment Ten-week-old female and male BALB/c mice (The Jackson

550 Laboratory, Bar Harbor, ME, USA) housed in the animal research facility of the UTMB

551 (Galveston, Texas) were used for these studies. Randomly selected groups of mice (50\% $\hat{\sigma}$ and

$55250 \%$ \%) were challenged via intranasal (i.n) route with RSV (106 PFU per mice) in $60 \mu$ of

553 phosphate-buffered saline solution (PBS; pH: 7.4) under mild isoflurane anesthesia. TH5487

554 (SelleckChem; 30 mg per kg, in a $200 \mu \mathrm{L}$ volume of solvent (5\% DMSO, 10\% Tween 80 in

555 saline) was administered via the peritoneal route $1 \mathrm{~h}$ before and every 8h after RSV challenge.

556 All experiments performed here are in accordance with the NIH Guide for Care and Use of

557 Experimental Animals and approved by the University of Texas Medical Branch (UTMB) Animal

558 Care and Use Committee (approval no. 0807044D).

559 Assessment of cellular ROS levels. Intracellular ROS levels were determined by using the

560 florigenic probe 5- (and-6)-chloromethyl-2'7'-dichlorodihydrofluorescein diacetate acetyl ester

561 (CM-H2DCFDA; Invitrogen, Eugene, OR). In brief, cells were mock- or RSV-infected and loaded

562 with $10 \mu \mathrm{M} \mathrm{CM}-\mathrm{H} 2 \mathrm{DCF}-\mathrm{DA}$ at $37^{\circ} \mathrm{C}$ for 10 minutes. Cells were then washed with PBS and lysed

563 (50 mM Tris- $\mathrm{HCl}, \mathrm{pH}$ 7.5, $150 \mathrm{mM} \mathrm{NaCl}, 1 \mathrm{mM}$ EDTA, 1 mM EGTA, 1\% NP-40), then clarified

564 by centrifugation. Changes in DCF fluorescence in supernatant fluids were determined by using

565 a Synergy H1 Hybrid Multi-Mode Reader (BioTek) with excitation / emission at $485 \mathrm{~nm} / 535$

$566 \mathrm{~nm}$. Results are expressed as change in fluorescence units (FU).

567 Gene specific siRNA depletion. Triplicate cultures of hSAEC or A549 cells were transfected

568 with siRNA targeting the hOGG1 (Dharmacon, L-005147-00-0020), hMTH1 (Dharmacon, L-

569 005218-00-0020), hNEIL1 (Dharmacon, L-008327-00-0020) mRNAs or with universal siRNA

570 negative control (Dharmacon, D-001810-01-20) using Lipofectamine RNA iMAX transfection 
571 reagent (13-778-075, Invitrogen, Carlsbad, CA, USA). The efficiency of gene silencing was

572 determined by qRT-PCR and Western blot analysis. Sequences of primers are listed in Table 2.

573

574

575

576

577

578

579

580

581

582

583

584

585

586

587

588

589

590

591

592

593

594

595

596

597

598

599

600

601

602

603

Transgenic over expression of OGG1. OGG1 deleted hSAECs were transfected with FLAGtagged OGG1. Sequence fidelity of OGG1 was determined in Molecular Genomic Facility of UTMB (https://www.utmb.edu/ mgf/next-generation-sequencing-core). One $\mu$ g plasmid per 35 mm dish (800,000 cell) were transfected using Lipofectamine 3000 as described in manufacturer instruction. Twenty-four h later, cells were mock or infected with RSV ( $3 \mathrm{MOI}$ per cells) for various lengths of time $(0,12,24,36 \mathrm{~h})$. At the time of infection, expression of (FLAG)OGG1 was determined by qRT-PCR using primer sets (Table 1) and Western blot analysis (Abcam, ab135940).

M2-1 Expression and Purification. An optimized sequence of RSV M2-1 gene fused with a Nterminal Histidine (His) tag was cloned into the expression vector pET28a and confirmed by sequencing (Creative Biogene Laboratories, Shirley, NY). Protein (His-M2-1) production was performed essentially as described previously ${ }^{54,58}$. Briefly, the E. coli BL21 (DE3) Star (ThermoFisher) was transformed with the expression vector pET28a-His-M2-1 and cultured in LB medium in the presence of $50 \mu \mathrm{g} / \mathrm{mL}$ kanamycin to an absorbance of $0.5-0.7$ at $600 \mathrm{~nm}$ before inducing His-tagged M2-1 expression with $0.5 \mathrm{mM} \mathrm{IPTG}, 50 \mu \mathrm{M} \mathrm{ZnSO}{ }_{4}$ for 3 hours at $37^{\circ} \mathrm{C}$. Harvested cells were flash-frozen in liquid nitrogen and stored at $-80^{\circ} \mathrm{C}$. For purification of His-M2-1, the frozen cell paste $(15 \mathrm{~g})$ with the overexpressed protein was re-suspended at $4^{\circ} \mathrm{C}$ in buffer $\mathrm{A}$ (50 mM Tris- $\mathrm{HCl} \mathrm{pH} \mathrm{7.4,} 300 \mathrm{mM} \mathrm{NaCl}, 1 \mathrm{mM} \beta$-mercaptoethanol) with one complete EDTA-free protease inhibitor tablet. The re-suspended cells were disrupted by three passes through a micro fluidizer (15,000 PSI, Microfluidics, Newton, MA). The cell debris were removed by centrifugation at $18,000 \mathrm{rpm}$ at $4^{\circ} \mathrm{C}$ for 45 minutes. Clarified supernatant was filtered through a $0.22-\mu \mathrm{m}$ filter (Millipore, Burlington, MA), loaded onto a $5 \mathrm{~mL}$ nickel HisTrap HP (GE Healthcare, Piscataway, NJ) fast-flow column previously equilibrated in buffer A. The column was first washed with buffer A containing $20 \mathrm{mM}$ imidazole, and then with high salt buffer (buffer $A$ with $1 \mathrm{M} \mathrm{NaCl}$ ) to remove loosely-bound nucleic acids from the protein. Elution of His-M2-1 from the His-Trap column was performed by a linear gradient of $50-1000 \mathrm{mM}$ imidazole in buffer $A$. The fractions containing M2-1 were combined, diluted with buffer A containing $50 \mathrm{mM} \mathrm{NaCl}$, and further purified by cation-exchange Hi-Trap SP column (GE Healthcare, Piscataway, NJ) using a linear gradient of 50-1000 mM NaCl that further removed nucleic acids. The purified His-M2-1 recombinant protein was exchanged into the low salt buffer 
604

605

606

607

608

609

610

611

612

613

614

615

616

617

618

619

620

621

622

623

624

625

626

627

628

629

630

631

632

633

634

635

636

(20 mM Tris- $\mathrm{HCl} \mathrm{pH} \mathrm{7.4,} 150 \mathrm{mM} \mathrm{NaCl}$ ) and its purity was confirmed by spectrophotometry (OD $260 \mathrm{~nm} / 280 \mathrm{~nm}$ ratio) and Western blot analysis.

RNA extraction and qRT-PCR. Total RNAs were extracted using RNeasy Mini kit (Qiagen) according to the manufacturer's instructions. Crude RNAs were DNasel-treated and loaded onto an RNeasy column and subjected to washes with RW1 and RPE buffers. RNAs were eluted with the RNase-free water included in the kit. In specific experiments, mRNAs were isolated using a magnetic mRNA isolation kit (NEB, S1550S). The RNA concentration was determined spectrophotometrically on an Epoch Take-3 ${ }^{\text {TM }}$ system (BioTek, Winooski, VT) using Gen5 v2.01 software. The quality of the RNAs were confirmed via the $260 / 280 \mathrm{~nm}$ ratio (varied between 1.9 -2.0). $1000 \mathrm{ng}$ total RNA was used to generate cDNA with either oligo-dT or random hexamer primers (Takara, Item \#: RR037A). qPCR was performed using specific primers for RSV NS1, $\mathrm{N}, \mathrm{G}, \mathrm{F}$ and $\mathrm{L}$ mRNA. The level of RSV genome was determined using primer pairs amplifying inter-genome (upstream from the attachment glycoprotein $\mathrm{G}$ ) and the 3'-end of $\mathrm{G}$ gene (genome coordinates 3' c). As internal control, cellular GAPDH was used (sequence of primers are listed in Table 1). Changes in mRNA and genomic RNA levels were expressed as fold increases over levels at $2 \mathrm{~h}$ (after adsorption) in infected cells using the $2^{-\Delta \Delta \mathrm{Ct}}$ method.

Co-Immunoprecipitation (Co-IP) and Western blot analysis. Parallel cultures of FLAGtagged OGG1 expressing cells $\left(1 \times 10^{7} \mathrm{hSAEC}\right)$ were RSV- $(\mathrm{MOI}=3)$ or mock-infected, and $24 \mathrm{~h}$ later cells were lysed using cell lysis buffer (Cell Signaling Technology; Item \#: 9803; 50 mM Tris-HCl, pH 7.5, $150 \mathrm{mM} \mathrm{NaCl}, 1$ mM EDTA, 1 mM EGTA, 1\% Nonidet P-40) containing 2.5 $\mathrm{mM}$ sodium pyrophosphate, $1 \mathrm{mM}$ glycerophosphate and protease inhibitors (1 mM Na3VO4, 1 $\mathrm{mM} \mathrm{NaF}$, and $20 \mu \mathrm{g} / \mathrm{mL}$ aprotinin, leupeptin, phenylmethanesulfonyl fluoride). Cell lysates were clarified (14,000 $\mathrm{g}$ at $4^{\circ} \mathrm{C}$ for $30 \mathrm{~min}$ ) and then incubated with Protein $\mathrm{G}$ beads (Millipore Sigma, Item \#: LSKMAGAG10) coupled to anti-FLAG antibody (Abcam, ab205606) for $3 \mathrm{~h}$ at $4^{\circ} \mathrm{C}$ with rotation. Cell lysates were also incubated with uncoupled beads or antibody to a control lgG (Santa Cruz). Beads were washed 3 times in PBS, and the proteins were eluted by boiling in $1 \mathrm{x}$ loading buffer. Eluted proteins were separated by SDS-PAGE electrophoresis, and western blot analysis was performed to detect immunoprecipitated RSV proteins. Proteins were transferred into nitrocellulose membranes, blocked with $5 \%$ non-fat dry milk in TBS-T (20 mM Tris base, $500 \mathrm{mM} \mathrm{NaCl}$, and $0.05 \%$ Tween-20, $\mathrm{pH}$ 7.5), and then incubated for $3 \mathrm{~h}$ with mouse anti-RSV Nucleoprotein monoclonal (Abcam, ab94806), mouse anti-RSV M2-1 monoclonal (Abcam, ab94805) and subsequently with horseradish peroxidase-conjugated secondary antibody 
(1:4000 dilution; SouthernBiotech, Birmingham, AL, USA). The signals were detected using the ECL Plus chemiluminescent detection system (Bio-Rad, Item \#: 1705061). Even loading was confirmed by probing membranes with GAPDH antibody (Item \#: 14C10; Cell Signaling Technology).

Microscopic Imaging. hSAECs were plated on collagen pre-treated cover glasses (Roche Applied Sciences) and infected with RSV $(\mathrm{MOI}=1)$ or mock-infected for increasing lengths of time. Cells were fixed with acetone : methanol (1:1) for $20 \mathrm{~min}$ at room temperature, dried and stored. At the time of use, cells were rinsed and permeabilized using $0.1 \%(w / v)$ Triton-X-100 diluted in phosphate-buffered saline (PBST) for $5 \mathrm{~min}$. Cells were incubated with $1 \%$ BSA for 1 $\mathrm{h}$ at room temperature and primary antibodies were added to individual slides at a dilution recommended by the manufacturer or determined in preliminary studies (1 to $100 ; 1$ to 300 ) in PBST for $1 \mathrm{~h}$ at $37^{\circ} \mathrm{C}$. Abs were specific to OGG1 (Novus, NB100-106), 8-oxo(r)Gua (Millipore Sigma, MAB3560), RSV M2-1 (Abcam, ab94805), anti-nucleoprotein (Abcam, ab94806), antiNitro tyrosine (Millipore Sigma, 06-284), and anti-2,4-Dinitrophenylhydrazine (DNP, Abcam, ab178020). After washing cells 3 times in PBST, secondary antibody conjugated to Alexa Fluor 488 (goat anti-rabbit) or Alexa Fluor 594 (goat anti-mouse) was added for $1 \mathrm{~h}$ at $37^{\circ} \mathrm{C}$. After washing in PBST (3-times), cells were dried and mounted with Vecta shield/DAPI, 4'6diamidino-2-phenylindole hydrochloride (Vector Laboratories, CA). Over 30 randomly selected fields of view per sample were photographed using a WHN10×/22 eyepiece and a $60 \times$ objective (field of view is $1.1 \mathrm{~mm}$, and camera correction is 0.63 ) on an OLYMPUS Microscope System BX53P.

Proximity ligation assay (PLA). hSAECs plated on collagen-treated coverslip slides were infected with RSV (1 MOI) for $24 \mathrm{~h}$ and fixed with $4 \%$ paraformaldehyde, and $0.1 \%(\mathrm{w} / \mathrm{v})$ permeabilized Triton-X-100 diluted in phosphate-buffered saline (PBST) for 5 min. After washing in PBS, Duolink $\circledast$ blocking solution was added for $1 \mathrm{~h}$ at room temperature and PLA was performed using the Duolink PLA kit (OLink Bioscience, catalog no. LNK-92101-KI01) per the manufacturer's instructions and as we described previously ${ }^{56}$. In brief, cells were incubated with the Ab to OGG1 (Novus, NB100-106) and RSV M2-1 (Abcam, ab94805) primary antibodies for $1 \mathrm{~h}$ at $37^{\circ} \mathrm{C}$. Cells were washed twice with $1 \mathrm{X}$ wash buffer A (Millipore Sigma, DUO82049), and secondary antibody conjugated MINUS and PLUS probes were added for $1 \mathrm{~h}$ at $37^{\circ} \mathrm{C}$. After washing with $1 \mathrm{x}$ buffer $\mathrm{A}$, ligation mix was added for $30 \mathrm{~min}$ at $37^{\circ} \mathrm{C}$, and then amplification mix was added to each sample for $100 \mathrm{~min}$ at $37^{\circ} \mathrm{C}$. After washing with buffer B (Millipore Sigma, 
669

670

671

672

673

674

675

676

677

678

679

680

681

682

683

684

685

686

687

688

689

690

691

692

693

694

695

696

697

698

699

700

Item \# DUO82049) cells were mounted with mounting medium (Ibidi Inc, Fitchburg, WI, Item \# 19-06-14). Cells were photographed using a WHN10x/22 eyepiece and a $60 \times$ objective (field of view is $1.1 \mathrm{~mm}$, and camera correction is 0.63) on an OLYMPUS Microscope System BX53P.

Measurement of co-localization: The measurement of co-localization between fluorophores (Alexa 594 (red) vs Alexa 488 (green) was performed using the software Image J v1.51, which calculates co-localization based on intensity of the individual fluorophore pixel (Pearson correlation coefficient) ${ }^{57}$.

RNA-protein cross-linking immunoprecipitation (RNA-IP). RNA-IP assays were performed as described by "CLIP-sequencing guidelines and practices" of the ENCODE and modENCODE consortia (http://encodeproject.org/ENCODE/) using slight modifications. Briefly, FLAG-tagged OGG1-transfected cells were infected with RSV (MOI = 1) for $24 \mathrm{~h}, \mathrm{RNA} /$ protein were crosslinked with $1 \%$ paraformaldehyde, and cells were harvested using 1 x lysis buffer containing iron chelator desferioxamine ${ }^{41}$ (DFO, Millipore Sigma, Item \#: 252750). RNA-protein complexes were immunoprecipitated using RNA-IP KIT (Active Motif, Item \#: 53024) with "ChIP" quality antibodies (Abs) [anti-FLAG Ab, Item \#: F1804, Sigma Millipore (Cambridge, MA); RSV M2-1 (Abcam, ab94805); IgG (Santa Cruz, sc-3887)]. The precipitates were washed three times, decross-linked and subjected to qRT-PCR. qRT-PCR reactions were performed in triplicate using SYBR Green PCR Master Mix (Bio-Rad) in a CFX 96 real-time PCR detection system (Bio Rad). The sequence of primers is listed in Table 1. qPCR data were normalized using the fold enrichment method. In brief, this method is based on the assumption that the IP using a specific $\mathrm{Ab}$ contains both specific signal and background signal, whereas the IP of a negative control$\lg G$ represents the background only. The background was subtracted from the signal, and the remaining value corresponded to the net pull-down of a specific RNA by a given specific $A b$ normalized to both total RNA and nonspecific (negative control) IP.

Electrophoretic mobility shift assay (EMSA). To evaluate OGG1 and/or M2-1 binding ssRNA or dsRNA with or without oxidative modification to Gua, EMSA was performed. RNA oligos (Table 3) were synthetized by Integrated DNA Technologies (IDT, Coralville, IA). For each reaction, 8 pmol 5'-Cy5-labeled probe was incubated with increasing concentrations $(0,2,4,8$ pmol) of OGG1 (ProSpec, Item \#: ENZ-253,) or M2-1 (made as described above) in a total volume of $10 \mu \mathrm{l}$ containing $10 \mathrm{mM}$ Tris- $\mathrm{HCl}$ (pH 8.0), $100 \mathrm{mM} \mathrm{NaCl}, 5 \mathrm{mM}$ EDTA, $1 \mathrm{mM}$ DTT. Binding assays were performed on ice for various lengths of time ( 1 to $30 \mathrm{~min}$ ). For competition assays, OGG1 was incubated with RNA for $15 \mathrm{~min}$, then M2-1 was added for increasing lengths 
701

702

703

704

705

706

707

708

709

710

711

712

713

714

715

716

717

718

719

720

721

722

723

724

725

726

727

728

729

730

731

732

733

of time (1 to $30 \mathrm{~min})$. For hybridization-coupled EMSA, mRNAs were isolated from RSV (0.1 MOI, 48 h) infected cells using Magnetic mRNA Isolation Kit (NEB, Item \#: S1550). One $\mu \mathrm{g}$ mRNA was mixed with $100 \mathrm{fmol}$ Cy5-labeled probe in hybridization buffer (100 mM Tris (PH 8.0), $50 \mathrm{mM} \mathrm{NaCl}, 1 \mathrm{mM}$ EDTA) and denatured at $95^{\circ} \mathrm{C}$ for $5 \mathrm{~min}$ and allowed to hybridize overnight. Mung bean nuclease (Item \#: M0250S, NEB) was used to digest the single-stranded un-hybridized probes and mRNAs at $30^{\circ} \mathrm{C}$ for $30 \mathrm{~min}$. 8-oxo(r)Gua-containing hybrids were identified by adding OGG1 ( $8 \mathrm{pmol}$ ) for $30 \mathrm{~min}$ on ice. Protein-DNA complexes were resolved on a $6 \%$ DNA retardation gel (Invitrogen, Item \# EC6365BOX) in $0.25 \times$ TBE buffer and visualized using Amersham ${ }^{\mathrm{TM}}$ Imager 680 (Global Life Sci. Sol. Marlborough, MA). Band intensities were quantified using Image J v1.51 (U. S. NIH, Bethesda, Maryland, USA).

Oligonucleotide excision assay. OGG1 glycosylase activity on 8-oxoGua containing dsRNA or DNA was assed as described previously ${ }^{53}$. The sequence of DNA probe: /5'-Cy5/-dAdGdAdG dAdAdGdAdAdGdAdAdGdAdAdGdAdA/8oxodG/dAdGdAdTdGdGdGdTdTdA dTdTdCdGdA dAdCdTdAdGdC-3'). Sequence of RNA probe is /5'-Cy5/-rCrArArArArUrGrGrArGrGrU rUrAr ArUrArU/8oxorG/rGrGrArArArUrGrArUrGrGrArA-3'). In brief, 100 fmol Cy5 labeled probes (Integrated DNA Technologies, Coralville, IO) were incubated with increasing concentrations (0.5, 1, or 2 pmol, Item \#: ENZ-253, ProSpec) of OGG1 in $10 \mu \mathrm{L}$ digestion buffer (10 mM of Tris$\mathrm{HCl}(\mathrm{pH} 7.5), 10 \mathrm{mM}$ of $\mathrm{NaCl}, 1 \mathrm{mM}$ of EDTA, $1 \mathrm{mg} / \mathrm{mL}$ BSA, and $1 \mathrm{mM}$ of DTT). After incubation for $10 \mathrm{~min}$ at room temperature, the reaction was halted by adding $10 \mu \mathrm{L}$ loading buffer (containing $8 \mu \mathrm{L}$ of formamide, $2 \mu \mathrm{L}$ of $10 \mathrm{mM}$ of $\mathrm{NaOH}$ ) and heated for 5 minutes at $95^{\circ} \mathrm{C}$. The cleaved product was separated from the intact probe in a $15 \%$ polyacrylamide gel containing $8 \mathrm{M}$ urea in Tris-borate-EDTA buffer $(\mathrm{pH} 8.4)$. The separated bands were visualized by using Amersham ${ }^{\mathrm{TM}}$ Imager 680.

Cell viability assay. $\mathrm{RSV}(\mathrm{MOI}=1)$ and mock-infected cells were treated with increasing concentrations $(2.5,5,10,20 \mu \mathrm{M})$ of TH5487, TH2840, TH588, O8 or $0.4 \%$ DMSO after virus adsorption every $8 \mathrm{~h}$. Cell culture medium was harvested at $48 \mathrm{~h}$ to perform the colorimetric lactate dehydrogenase assay ( $\mathrm{LDH}$; Abcam, Item \# ab102526). The assay was performed using a 96-well plate according to the manufacturer's instruction. The output of triplicate samples was determined immediately (T1), $10 \mathrm{~min}$ (T2), $20 \mathrm{~min}$ (T3), and $30 \mathrm{~min}$ (T4) at OD $450 \mathrm{~nm}$ on a microplate reader (Synergy H1 Hybrid Multi-Mode Reader; BioTek) at $37^{\circ} \mathrm{C}$ protected from light. Amounts of $\mathrm{LDH}$ in the media were calculated using $\triangle \mathrm{A} 450 \mathrm{~nm}=(\mathrm{A} 2-\mathrm{A} 1)$ formula, where: $A 1$ is the sample reading at time $T 1 . A 2$ is the sample reading at time T2. LDH activity is expressed in nmol of $\mathrm{NADH}$ generated by $\mathrm{LDH}$ during the reaction time $(\triangle \mathrm{T}=\mathrm{T} 2-$ 
734

735

736

737

738

739

740

741

742

743

744

745

746

747

\begin{tabular}{|l|l|}
\hline hGAPDH & $\begin{array}{l}\text { Forward primer: 5'-ACATCGCTC AGACACCATG -3' } \\
\text { Reverse primer: 5'-TGTAGTTGAGGTCAATGAAGGG -3' }\end{array}$ \\
\hline RSV NS1 mRNA & $\begin{array}{l}\text { Forward primer: 5' - GCTTTGGCTAAGGCAGTGAT -3" } \\
\text { Reverse primer: 5'- TGGCATTGTTGTGAAATTGG -3' }\end{array}$ \\
\hline RSV N mRNA & $\begin{array}{l}\text { Forward primer: 5'- AAGGGATTTTTGCAGGATTGTTT -3' } \\
\text { Reverse primer: 5'- CTCCCCACCGTAACATCACTTG -3' }\end{array}$ \\
\hline RSV G mRNA & $\begin{array}{l}\text { Forward primer: 5'- CGGCAAACCACAAAGTCACA -3' } \\
\text { Reverse primer: 5'- TTCTTGATCTGGCTTGTTGCA -3' }\end{array}$ \\
\hline RSV F mRNA & $\begin{array}{l}\text { Forward primer: 5'- AA CAGATGTAAGCAGCTCCGTTATC -3' } \\
\text { Reverse primer: 5'- CGATTTTTATTGGATGCTGTACATTT -3' }\end{array}$ \\
\hline RSV L mRNA & $\begin{array}{l}\text { Forward primer: 5'- CACTCTACAAAACAAAAAGACACAATCA -3' } \\
\text { Reverse primer: 5'- AGGATGTTGCATTGAACACATT -3' }\end{array}$ \\
\hline RSV genomic RNA & $\begin{array}{l}\text { Forward primer: 5'- AGTCATAACA ATGAACTAGG -3' } \\
\text { Reverse primer: 5'- TTCTTGATCTGGCTTGTTGCA -3" }\end{array}$ \\
\hline hOGG1 & $\begin{array}{l}\text { Forward primer: 5'- GGCTCAACTGTATCACCACTGG -3' } \\
\text { Reverse primer: 5'-GGCGAT GTTGTTGTTGGAGGAAC -3' }\end{array}$ \\
\hline hNEIL1 & $\begin{array}{l}\text { Forward primer: 5'-GACAGAGGCAAGTGGCAAAGCA -3' } \\
\text { Reverse primer: 5'-GCCTCATTCACAAACTGGCTGG -3' }\end{array}$ \\
\hline hMTH1 & $\begin{array}{l}\text { Forward primer: 5'-GTCTTCTGCACAGACAGCATCC -3' } \\
\text { Reverse primer: 5'-CTGAAGCAGGAGTGGAAACCAG -3' }\end{array}$ \\
\hline
\end{tabular}

T1). $\mathrm{LDH}$ activity $=(\mathrm{Bl}(\Delta \mathrm{T} \times \mathrm{V})) \times \mathrm{D}=\mathrm{nmol} / \mathrm{min} / \mathrm{mL}, \mathrm{B}=$ amount of $\mathrm{NADH}$ in sample, calculated from standard curve (nmol). $\Delta \mathrm{T}=$ Reaction time (minutes). $\mathrm{V}=$ Original sample volume added into the reaction well $(\mathrm{mL})$. $\mathrm{D}=$ Sample dilution factor.

Statistical analysis. The data are expressed as the mean \pm SD. Results were analyzed for significant differences using unpaired, two-tailed Student's t-tests using Microsoft Excel procedures. Differences were considered significant at $p<0.05\left({ }^{*} p<0.05,{ }^{* *} p<0.01,{ }^{* *} p<0.001\right.$, $\left.{ }^{* * * *} \mathrm{p}<0.0001\right)$.

2

3

\section{Table 1. Primer sequences for PCR}




\begin{tabular}{|c|c|}
\hline $\begin{array}{l}\text { ON-TARGET plus Human MTH1 } \\
\text { (GeneID:4521) siRNA-SMART pool }\end{array}$ & $\begin{array}{l}\text { Target sequence1: 5'- GGGCAAAGUGCAAGAAGGA -3' } \\
\text { Target sequence2: 5'- GGAGAGCGGUCUGACAGUG -3' } \\
\text { Target sequence3: 5'- GAAAUUCCACGGGUACUUC -3' } \\
\text { Target sequence4: 5'- UGUUUGAGUUCGUGGGCGA -3' }\end{array}$ \\
\hline $\begin{array}{l}\text { ON-TARGET plus Human OGG1 } \\
\text { (GenelD:4968) siRNA-SMART pool }\end{array}$ & $\begin{array}{l}\text { Target sequence1: 5'- CGACAAGACCCCAUCGAAU -3' } \\
\text { Target sequence2: 5'- GGACAAUCUUUCCGGUGGA -3' } \\
\text { Target sequence3: 5'- GCUCAGAAAUUCCAAGGUG -3' } \\
\text { Target sequence4: 5'- UACCCUGGCUCAACUGUAU -3' }\end{array}$ \\
\hline $\begin{array}{l}\text { ON-TARGET plus Human NEIL1 } \\
\text { (GeneID:79661) siRNA-SMART pool }\end{array}$ & $\begin{array}{l}\text { Target sequence1: 5'- UACGAAACCUAGCGGAUAA -3' } \\
\text { Target sequence2: 5'- GACCAGAGGUUCUUCAAUG -3' } \\
\text { Target sequence3: 5'- UGACAUCCCAUCCUUGGAA -3' } \\
\text { Target sequence4: 5'- GGACCAAGCUGCAGAAUCC -3' }\end{array}$ \\
\hline ON-TARGET plus Non-targeting siRNA & 5'- UGGUUUACAUGUCGACUAA -3' \\
\hline
\end{tabular}

Table 3. Sequences for EMSA

\begin{tabular}{|l|l|}
\hline $\begin{array}{l}\text { RSV RNA probe - F } \\
\text { (genome sense) }\end{array}$ & 5'- UGCGUUGGUCCUUGUUUUUGGACAUGUUUGCAUUUGCCCC-3' \\
\hline $\begin{array}{l}\text { RSV RNA probe - R } \\
\text { (mRNA sense) }\end{array}$ & 5'-GGGGCAAAUGCAAACAUGUCCAAAAACAAGGACCAACGCA Cy5 -3' \\
\hline $\begin{array}{l}\text { RSV RNA probe - 8- } \\
\text { oxo(r)Gua - R (mRNA } \\
\text { sense) }\end{array}$ & 5'- GGGG*CAAAUGCAAACAUGUCCAAAAACAAGGACCAACGCA Cy5 -3' \\
\hline $\begin{array}{l}\text { DNA probe - F (positive } \\
\text { control) }\end{array}$ & 5'-AGAGAAGAAGAAGAA G*AGATGGGTTATTCGAACTAGC-3' \\
\hline $\begin{array}{l}\text { DNA probe - R (positive } \\
\text { control) }\end{array}$ & 5'- Cy5-GCTAGTTCGAATAACCCATCTCTTCTTCTTCTTCTCT-3' \\
\hline $\begin{array}{l}\text { RSV DNA probe - } \\
\text { intergenic (genome } \\
\text { sense) }\end{array}$ & 5'-Cy5 AATGTTATTGTTAGTCTTGATATCCTAGTTCATTGTTATG-3' \\
\hline $\begin{array}{l}\text { RSV DNA probe - } \\
\text { 5'UTR (genome sense) }\end{array}$ & 5'-Cy5 TGCGTTGGTCCTTGTTTTTGGACATGTTTGCATTTGCCCC-3' \\
\hline $\begin{array}{l}\text { RSV DNA probe - } \\
\text { 3'UTR (genome sense) }\end{array}$ & 5'-Cy5 TTTTTAAGTAACTACTGGCGTGGTGTGTTGGGTGGAGATG-3' \\
\hline
\end{tabular}


FIGURE 1
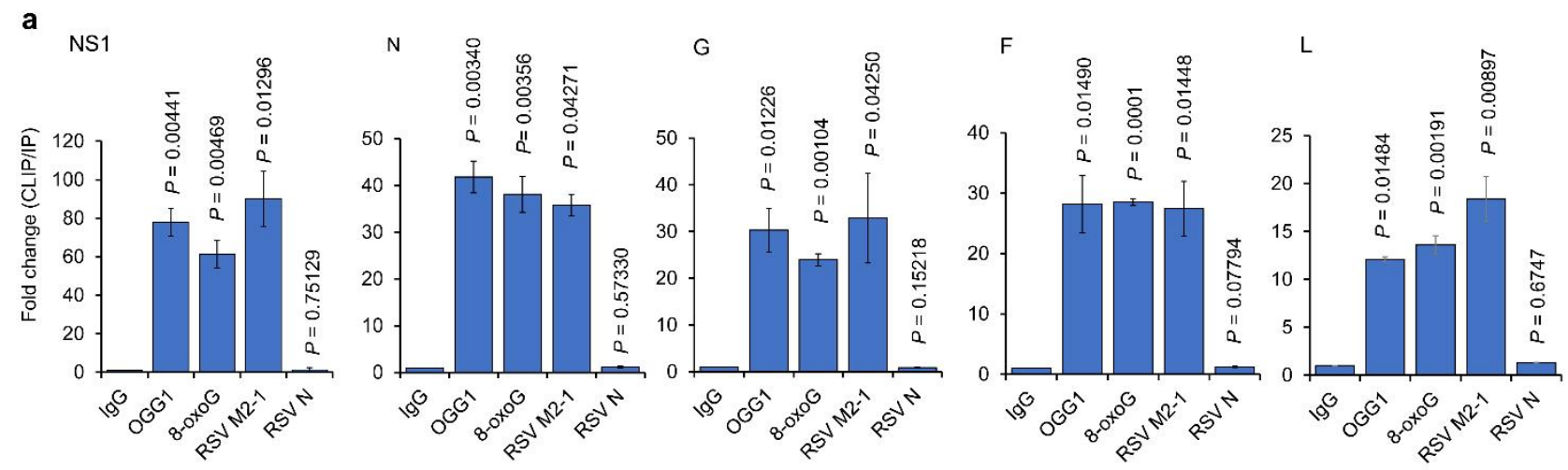

b
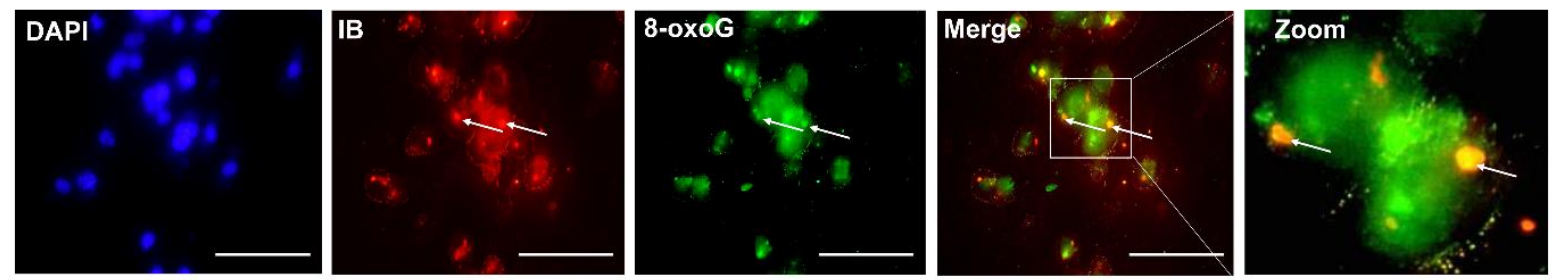

c
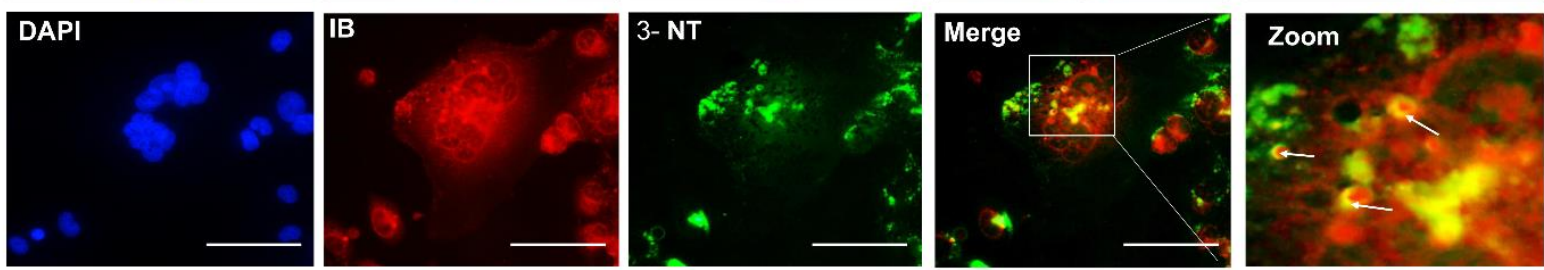

d
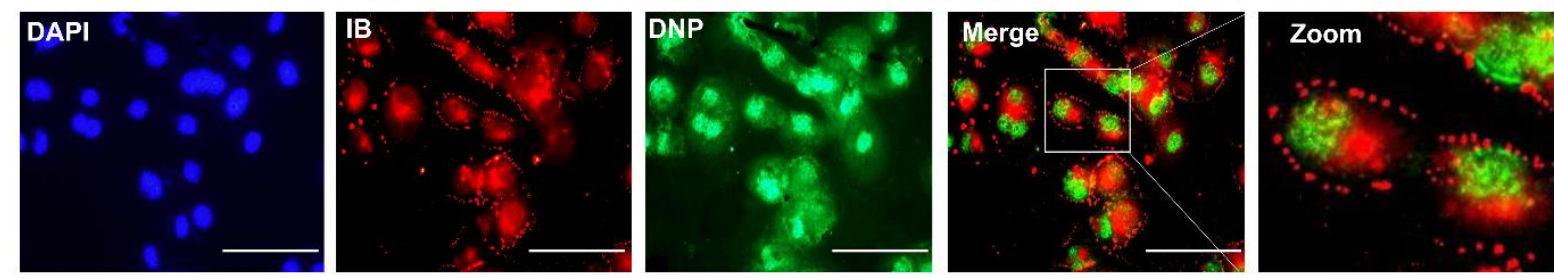

Oxidative modification to guanine nucleotide in RSV RNAs in the cytoplasmic inclusion bodies

a. Enrichment of OGG1 on viral RNAs and immunoprecipitation by antibody to 8-oxoGua.

Parallel cultures of OGG1 proficient hSAECs were infected with RSV (MOI = 1), protein-RNA complexes were cross-linked and samples IP-ed using antibodies to OGG1, M2-1 or N proteins. In parallel, RNAs were isolated and then IP-ed with 8-oxoGua-specific antibody. Levels of vRNA in immuno-precipitates were determined by qRT-PCR. The experiment was done three times $(n$ $763=3)$. b,c,d. Oxidatively modified RNAs co-localized with virus-induced cytoplasmic inclusion bodies. RSV infected hSAECs were fixed and immunochemically-stained with Abs to nucleoprotein $\mathrm{N}$ protein, 8-oxoGua, 3-nitrotyrosine or 2,4-Dinitrophenylhydrazine (protein carbonyls). Randomly 
767 selected fields were photographed using an OLYMPUS Microscope System BX53P, with a built768 in digital CCD color camera (DP73WDR). Magnification: 60X; scale bar: $40 \mu \mathrm{m}$. The

769 experiments were performed twice with two biological replicates each. IB, inclusion body; 3-NT, 770 3-Nitrotyrosine; DNP, Dinitrophenol, IBs, inclusion bodies, Abs, antibodies, IP-ed,

771 immunoprecipitated

772

773

774 
FIGURE 2
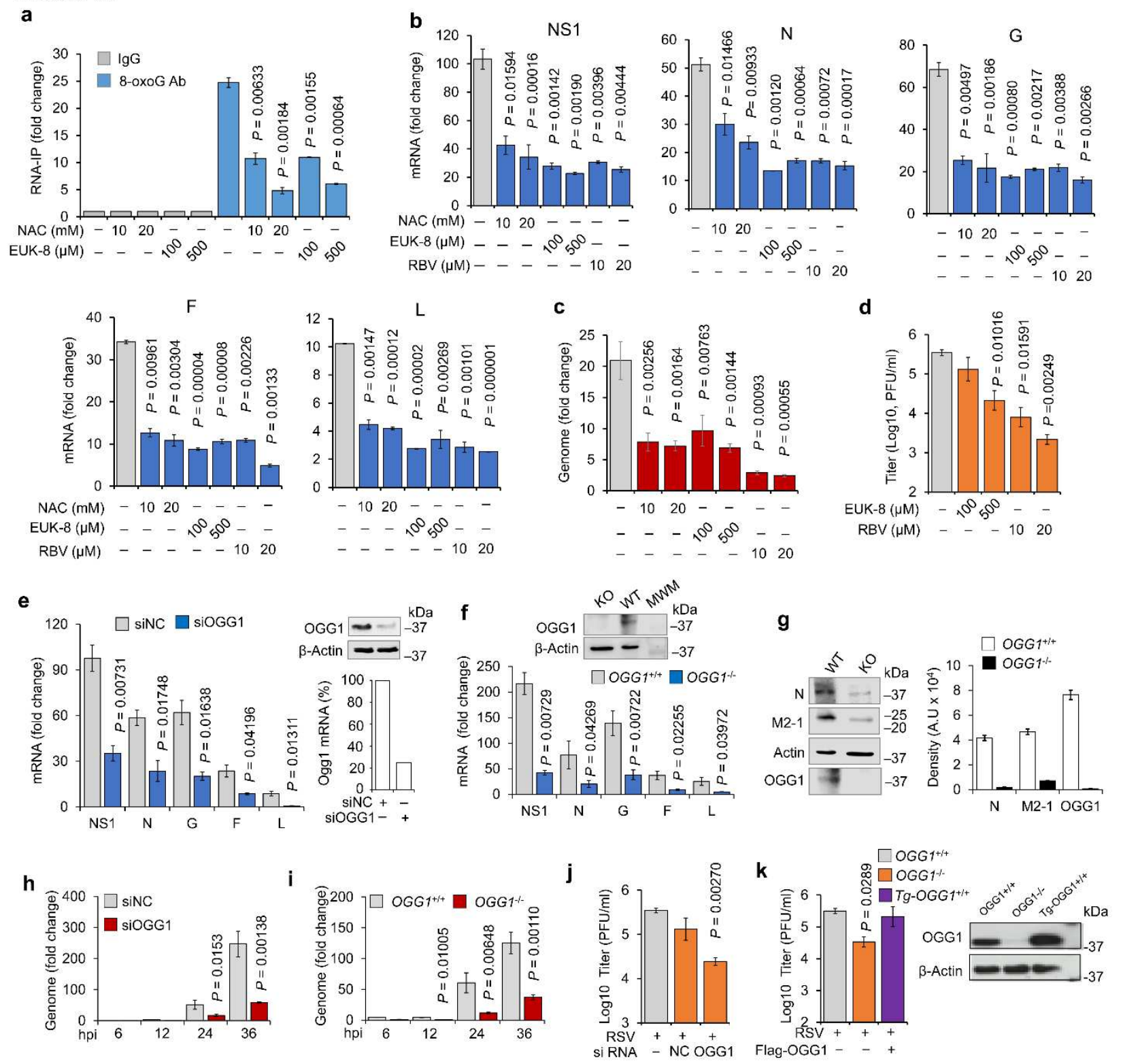

Decrease in Gua oxidation or silencing OGG1 expression lowers output of RSV infection

777 a Decrease in ROS levels by antioxidants lowers 8-oxo(r)Gua-containing mRNA coded by $\mathrm{G}$

778 gene. RSV-infected hSAEC were mock- or treated with NAC, EUK-8 (concentrations as

779 indicated). mRNAs were isolated, then immunoprecipitated by 8-oxoGua Ab. G mRNA levels in

780 immunoprecipitated RNAs were determined by qRT-PCR. Fold enrichment was calculated

781 relative to $\lg$ control $^{74}$.

782 b, c, d Decrease in oxidatively modified Gua levels in viral mRNAs lowers RSV output. RSV-

783 infected hSAECs were treated with AOs or with ribavirin (as control, concentrations as indicated

784 in Figs). Levels of mRNAs (b) and viral genome (c) were determined by qRT-PCR. RSV 
785 progeny (d) was determined by plaque assay. NS1, non-structural protein 1; N, nucleoprotein, 786 G, attachment glycoprotein G; F, fusion protein; L, viral RNA-dependent RNA polymerase.

787 e f $\mathbf{g}$ OGG1 silencing led to a decrease in viral mRNA and protein levels. OGG1-silenced

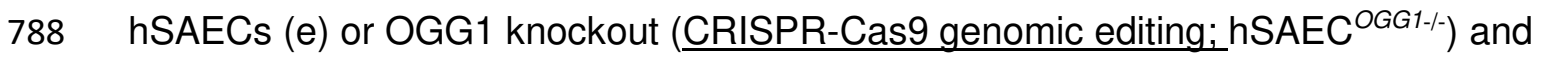

789 corresponding control (hSAECs and puromycin resistant hSAECs) cells (f) were infected with

790 RSV, and changes in mRNAs levels from NS1, N, G, F and L genes were determined by qRT-

791 PCR $(e, f)$. Protein levels of $\mathrm{N}$, and M2-1 were determined by Wb $(\mathrm{g})$. Inset to panel e and f:

792 OGG1 expression at RNA and protein levels. Right panel to g, quantification of band intensities $793(\mathrm{n}=3$; Image J v1.51). NS1, N, G, F and L, as in legend to b.

794 h i OGG1-silenced or knockout cells do not support RSV genome replication. OGG1 depleted

795 and knockout hSAECs (and corresponding controls, as in legend to e, f) were infected with RSV 796 and genome levels were determined by qRT-PCR.

797 j k Decreased yield of RSV in knockout hSAECs is restored by transgenic expression of OGG1. 798 (j) hSAEC transfected with control (NC) or siRNA to OGG1 were RSV-infected. (k)

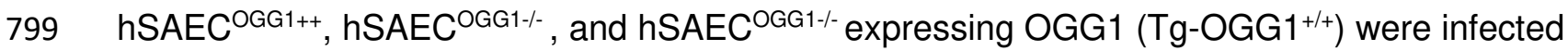

800 with RSV. Titers were determined by plaque assay. Right panel to k shows levels of OGG1. In

801 Figs b, c, d, e, f, g, j, k, $P$ values are of two biological replicates with two parallels.

802 
FIGURE 3
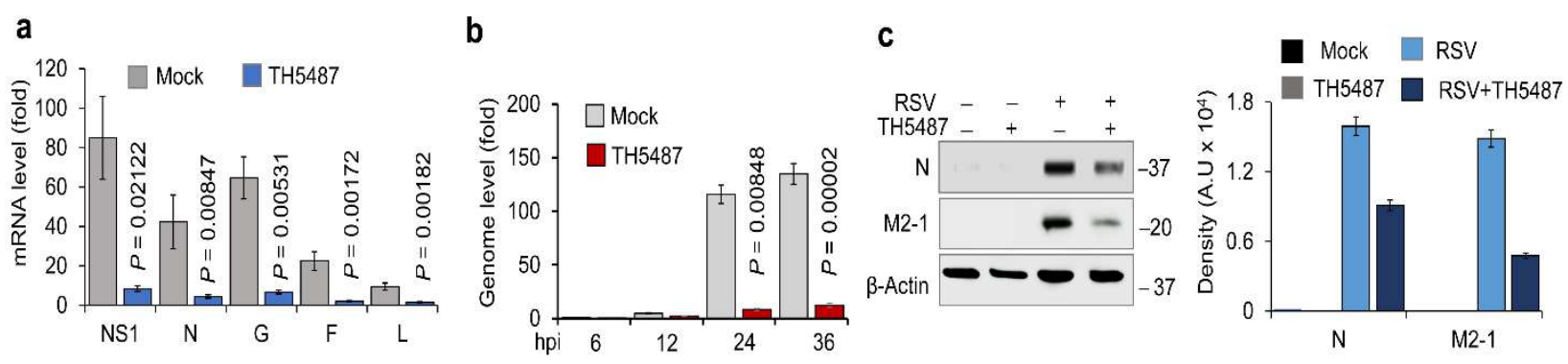

d
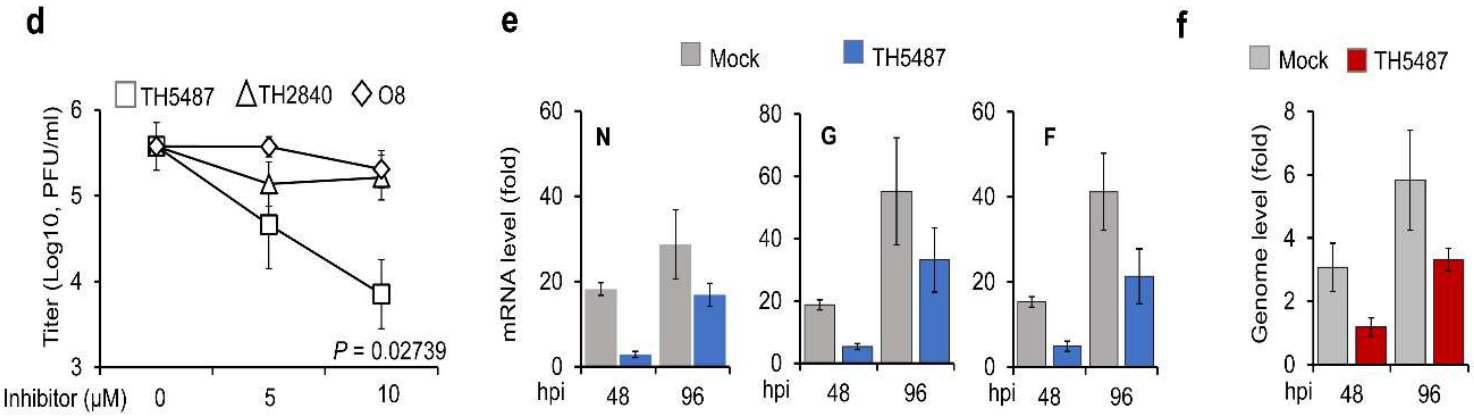

Inhibition of OGG1 binding to 8-OxO(r)Gua in viral RNA, lowers output of RSV infection in cultured cells and lungs

a b c Active site inhibitor of OGG1, TH5487 decreases viral mRNA, protein and genome levels. hSAECs were RSV-infected and mock- or TH5487-treated (10 $\mu \mathrm{M})$. mRNA and genome level $(a, b)$ and protein (c) levels were determined by qRT-PCR and Wb analysis, respectively. Right panel to c: band intensities (Image $\mathrm{J}$ v1.51). $P$ values are from two biological replicates with two parallels. TH5487, 4-(4-Bromo-2-oxo-3H-benzimidazol-1-yl)-N-(4- iodophenyl) piperidine-1carboxamide $^{49}$. RNA coding for NS1, non-structural protein 1; N, nucleoprotein, G, attachment

812 glycoprotein G; F, fusion protein and L, viral RNA-dependent RNA polymerase.

813 d OGG1 inhibitor decreases yield of RSV progeny. RSV-infected hSAECs were treated with

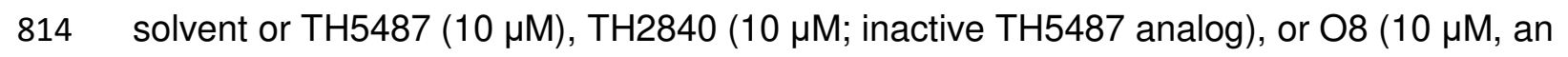

815 inhibitor of OGG1' glycosylase activity). RSV titer was determined by plaque assay $(n=3)$.

816 TH5487, as in a, TH2840, 4-(2-oxo-2,3-dihydro-1H-1,3-benzodiazol-1-yl)-N- phenylpiperidine-1-

817 carboxamide) $)^{49}$; O8, 3,4-Dichlorobenzo[b]thiophene-2-carbohydrazide ${ }^{50}$.

818 e f OGG1 agonist TH5487 decreases RSV replication in lungs. Mice $(n=6)$ were RSV-infected 819 by intrapulmonary challenge, and TH5487 was added via intraperitoneal route. At the indicated 820 time points, mRNAs levels from N, G, and F genes (e) as well as genome levels (f) were 821 determined by qRT-PCR. $P$ values are from one experiment with six replicates. hpi, hours post822 infection. N, G, F, as in legend to a. 


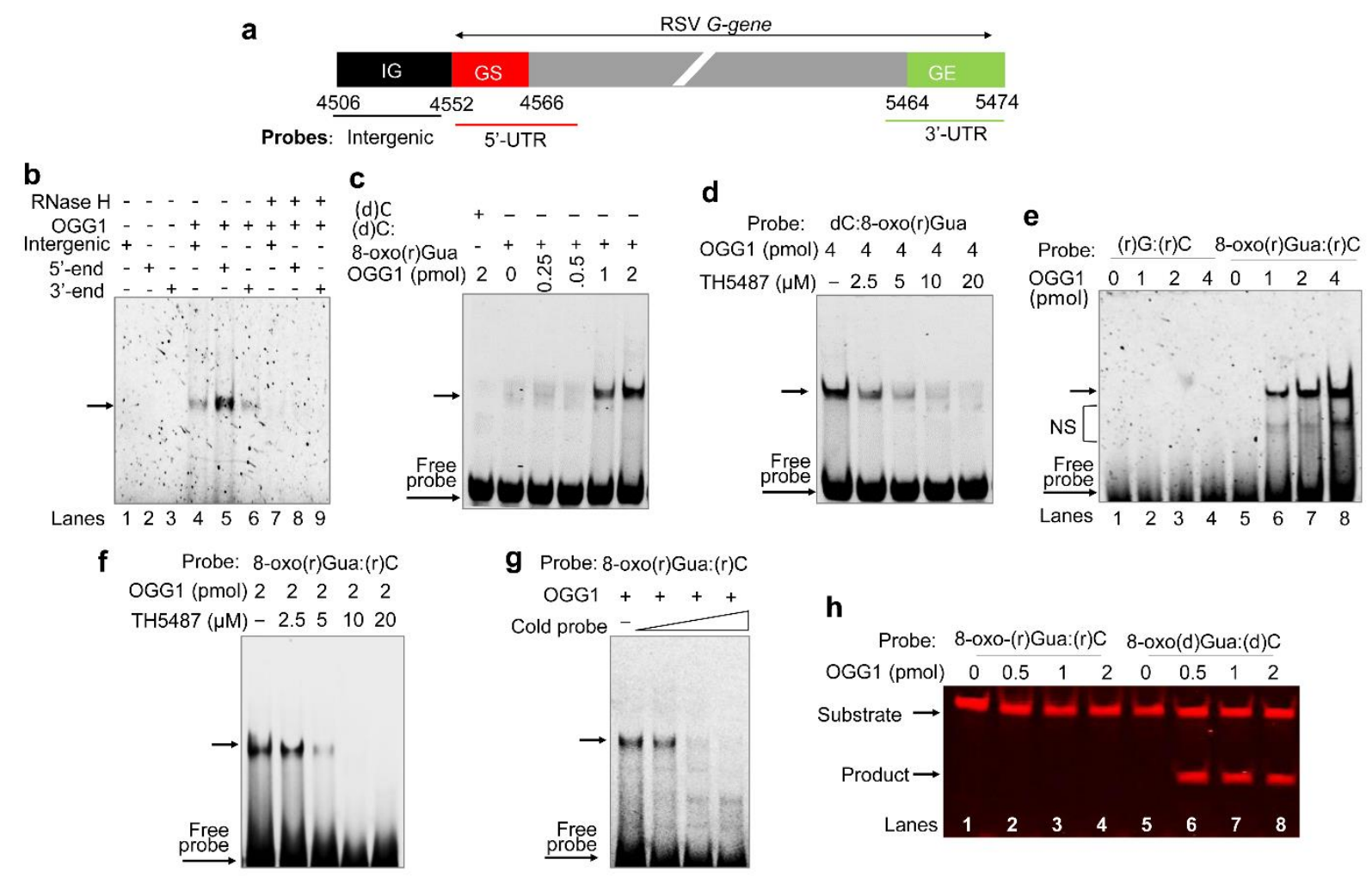

\section{5'-UTR of viral mRNAs are the hot-spot of Gua oxidation}

827 a Graphical depiction of G-gene and locations of sequences that served for probe design

828 (sequences are in Table 1).

829 b "Hot-spot" of 8-oxo(r)Gua generation is at the 5'-UTR of mRNA. RNAs isolated from infected

830 cells were mixed individually with single-stranded Cy5-labeled DNA probes (40 nt long)

831 complementary to genome, 5'-end (containing 5-UTR), 3'-end (containing 3-UTR) of mRNA

832 coding for G-protein and hybridized. Non-annealed sequences were removed by Mung bean

833 nuclease ${ }^{48}$. Cy5-DNA:8-oxo(r)Gua-containing vRNA hybrids were captured by OGG1 (lanes

$8344,5,6)$ by using EMSA. RNaseH-treatment degraded DNA:RNA hybrids (DNA:RNA hybrids, lane

$8357,8,9)$. There were no interaction between OGG1 and probes (lanes 1,2,3).

836 c d Concentration-dependent OGG1 binding (c) and its inhibition by TH5487 (d) to DNA:RNA

837 hybrids [(d)C:8-oxo(r)Gua-containing RNA].

838 e OGG1 binds only to RNA:RNA hybrid-containing 8-oxo(r)Gua (Lanes 6,7 and 8).

839 f TH5487 prevents binding of OGG1 to dsRNA containing 8-oxo(r)Gua [(r)C:8-oxo(r)Gua].

840 g OGG1 binding to labeled (Cy-5)dsRNA is competed out by "cold" dsRNA [(r)C:8-oxo(r)Gua)].

841 Arrow: RNA:RNA hybrid [(r)C:8-oxo(r)Gua]. In e,f,g OGG1 was incubated with (r)C:8-oxo(r)Gua)

842 RNA-RNA hybrids, and mixtures were subjected to EMSA. 
843 h Lack of OGG1' enzymatic activity(es) on dsRNA [8-oxo(r)Gua:(r)C]. Excision assays were

844 performed using 8-oxo(r/d)Gua-containing dsDNA and dsRNAs. Lanes 1-4 dsRNA [8-

845 oxo(r)Gua:(r)C]; Lanes 5-8: dsDNA [8-oxo(d)Gua:(d)C]. Representative images are shown in c,

$846 \mathrm{~d}, \mathrm{e}, \mathrm{f}, \mathrm{g}, \mathrm{h}(\mathrm{n}=3)$. In panel b to $\mathrm{h}$ images were captured by using Amersham ${ }^{\mathrm{TM}}$ Imager 680 .

847 Arrows: Cy5-DNA:vRNA hybrid containing 8-oxo(r)Gua.

848 
FIGURE 5
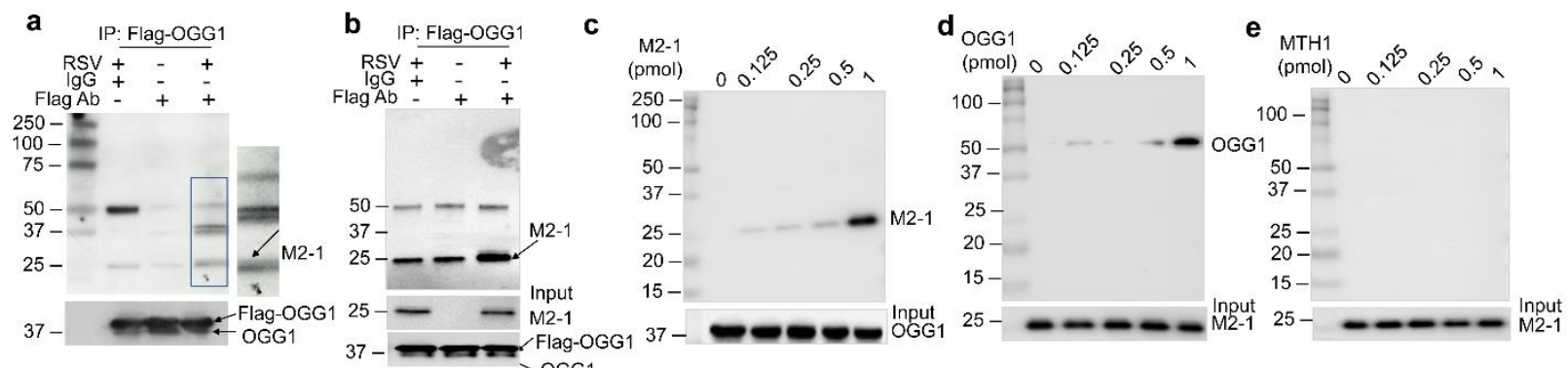

f Time (hpi)

Oh
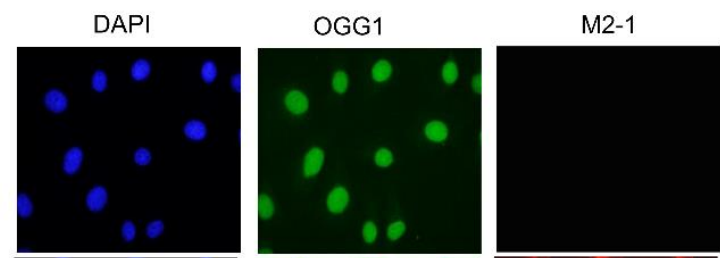

Merge
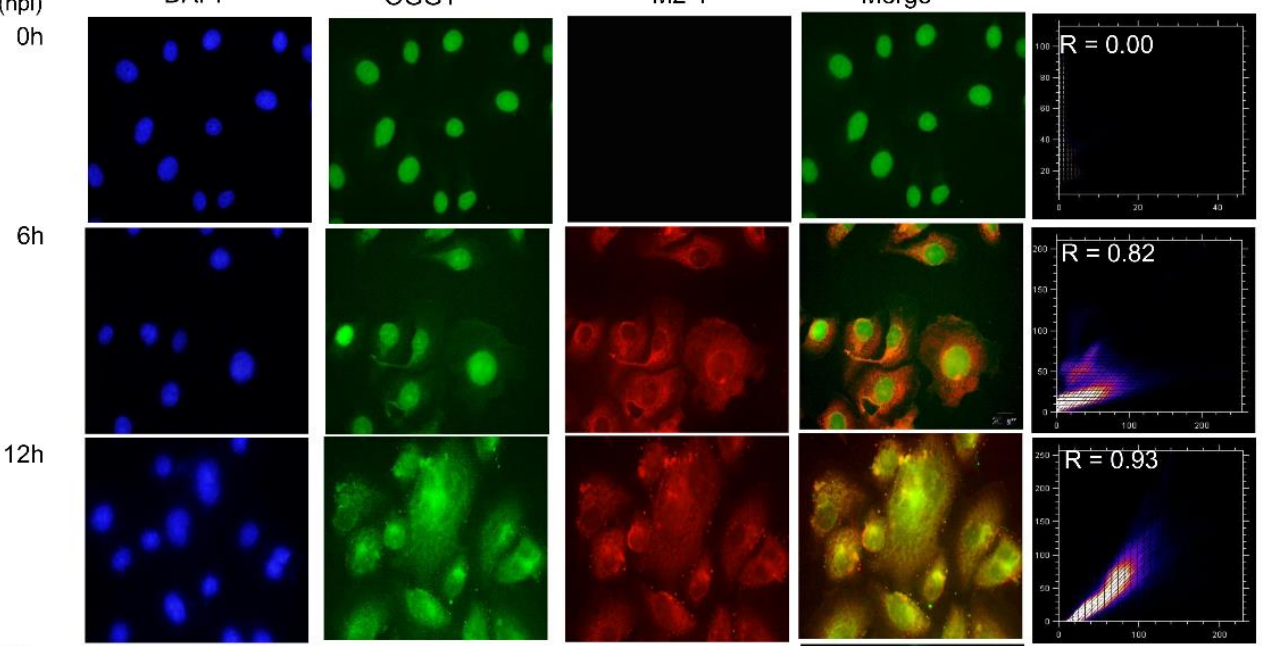

$12 \mathrm{~h}$
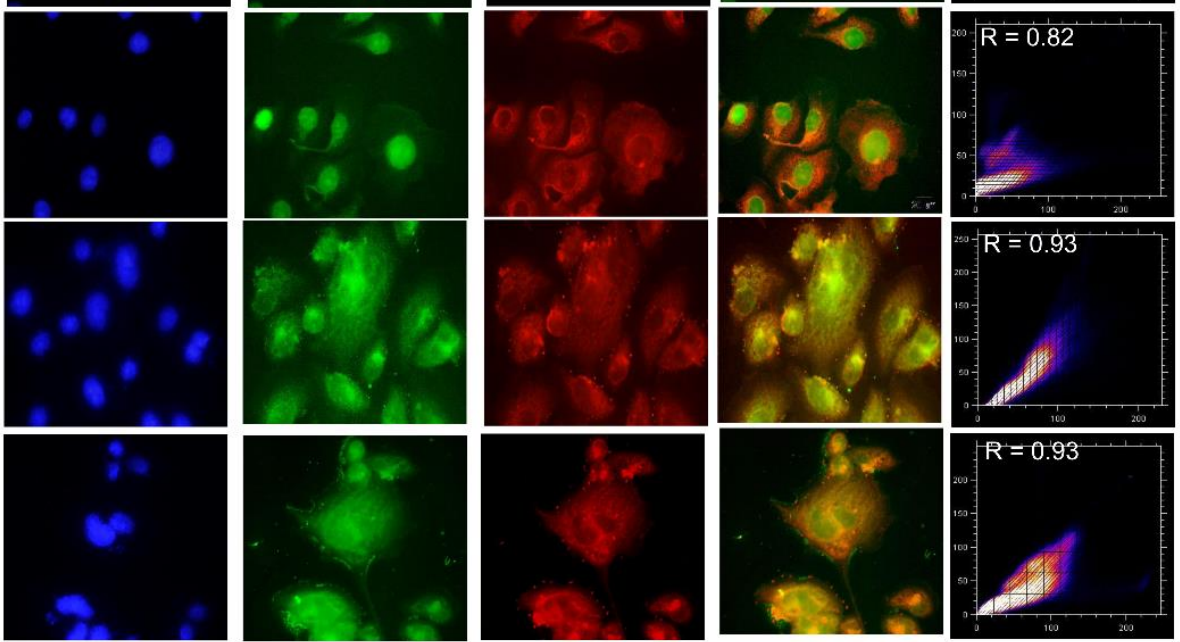

$24 \mathrm{~h}$

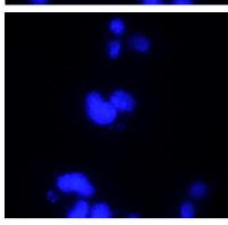

$36 \mathrm{~h}$
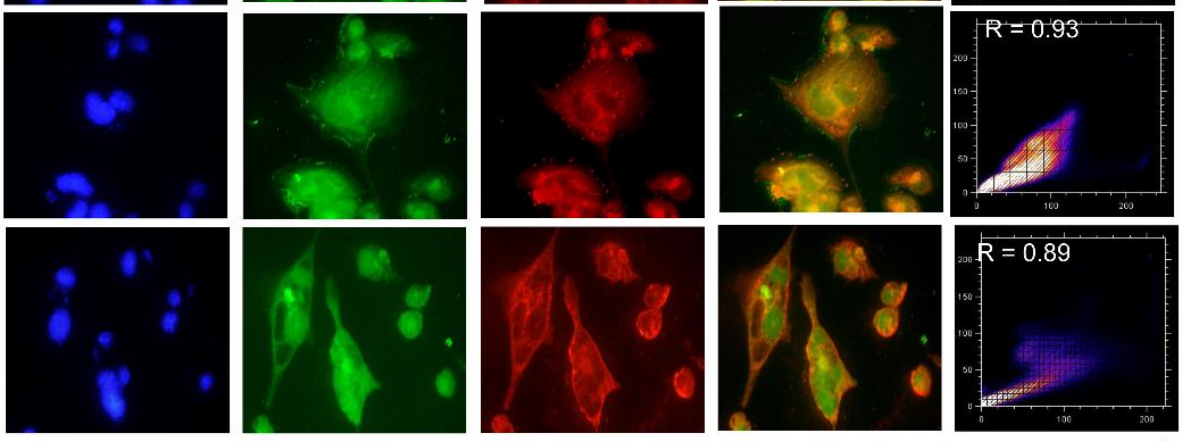

\section{g}

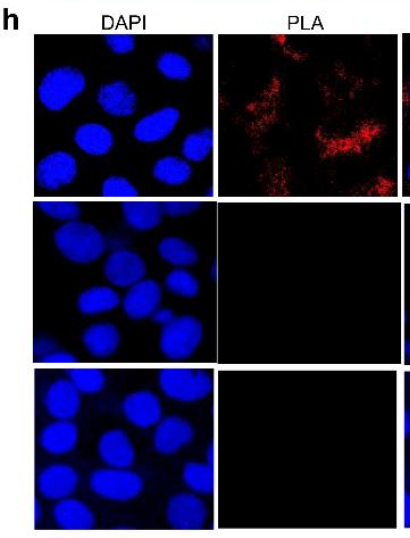

PLA

Merge

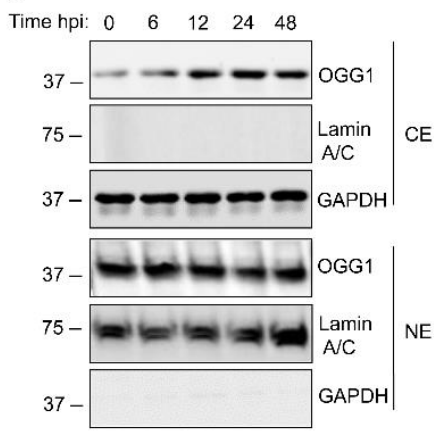


a b c d e Physical interaction of OGG1 with M2-1. FLAG-tagged OGG1-expressing cells were RSV-infected, lysed and lysates were subjected to IP using Ab to FLAG(OGG1). IP-ed proteins were immunoblotted by using polyclonal antibody to RSV proteins. Lower panel, expression of OGG1 in RSV-infected cells (a). Direct interaction between OGG1 and M2-1 proteins.

856 Equimolar GST-OGG1 and M2-1 were incubated and then immunoblotted using Abs to OGG1

857 or M2-1 (c, d). Lack of interaction between MTH1 and M2-1 proteins, shown in panel (e).

858 f Protein-protein interaction between OGG1 and M2-1 occur in IBs in cellulo. hSAECs were 859 RSV-infected for increasing length of time, fixed, then immuno-stained with Abs to OGG1 and

860 M2-1. Randomly selected fields were microscopically examined and photographed using an

861 OLYMPUS Microscope System BX53P with a built-in digital CCD color camera. Magnification:

$86260 X$; scale bar: $20 \mu \mathrm{m}$. Most-right column: Pearson fluorophores-moment correlation coefficient 863 (Alexa 594 and Alexa 488) was performed using Image J v1.51 software ${ }^{57}$.

864 g OGG1 accumulates in the cytoplasm of RSV-infected cells. RSV-infected hSAECs were 865 fractionated at the times indicated. Equal amounts of cytoplasmic (CE) and nuclear extract (NE) 866 were subjected to $\mathrm{Wb}$ analysis.

867 h Physical interaction between OGG1 and M2-1 as shown by proximity ligation assays (PLA). 868 RSV-infected cells were processed, and PLAs were performed. Cells were photographed as 869 described in panel f, g, h. Magnification: 60X; scale bar: $20 \mu \mathrm{m}$. Upper panels: PLA signals are 870 localized to cytoplasmic compartments of infected cells. PLA signals were absent when OGG1 871 (middle panels) or M2-1 (lower panels) specific primary antibodies were separately incubated 872 with cells and then the species-specific secondary antibodies conjugated to DNA

873 oligonucleotides were added.

874 i Physical interaction between OGG1 and M2-1 within IB as shown by PLA. RSV-infected cells 875 were incubated with Ab to OGG1 and/or M2-1, and ligated DNA oligonucleotides conjugated to 876 secondary Abs were amplified. Cells were photographed as described in panel f, g, h.

877 Magnification: 60X; scale bar: $10 \mu \mathrm{m}$.

878 
FIGURE 6
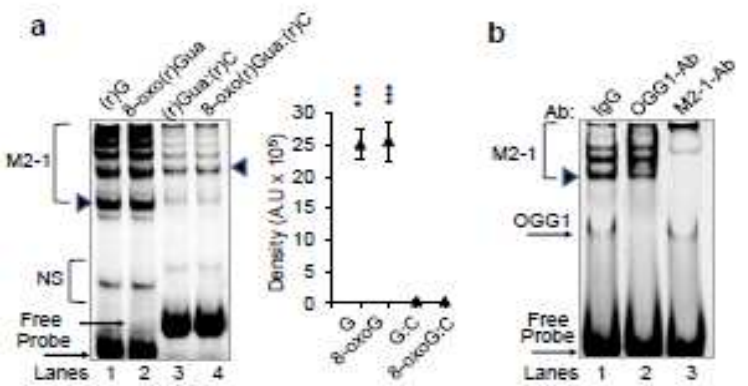

c
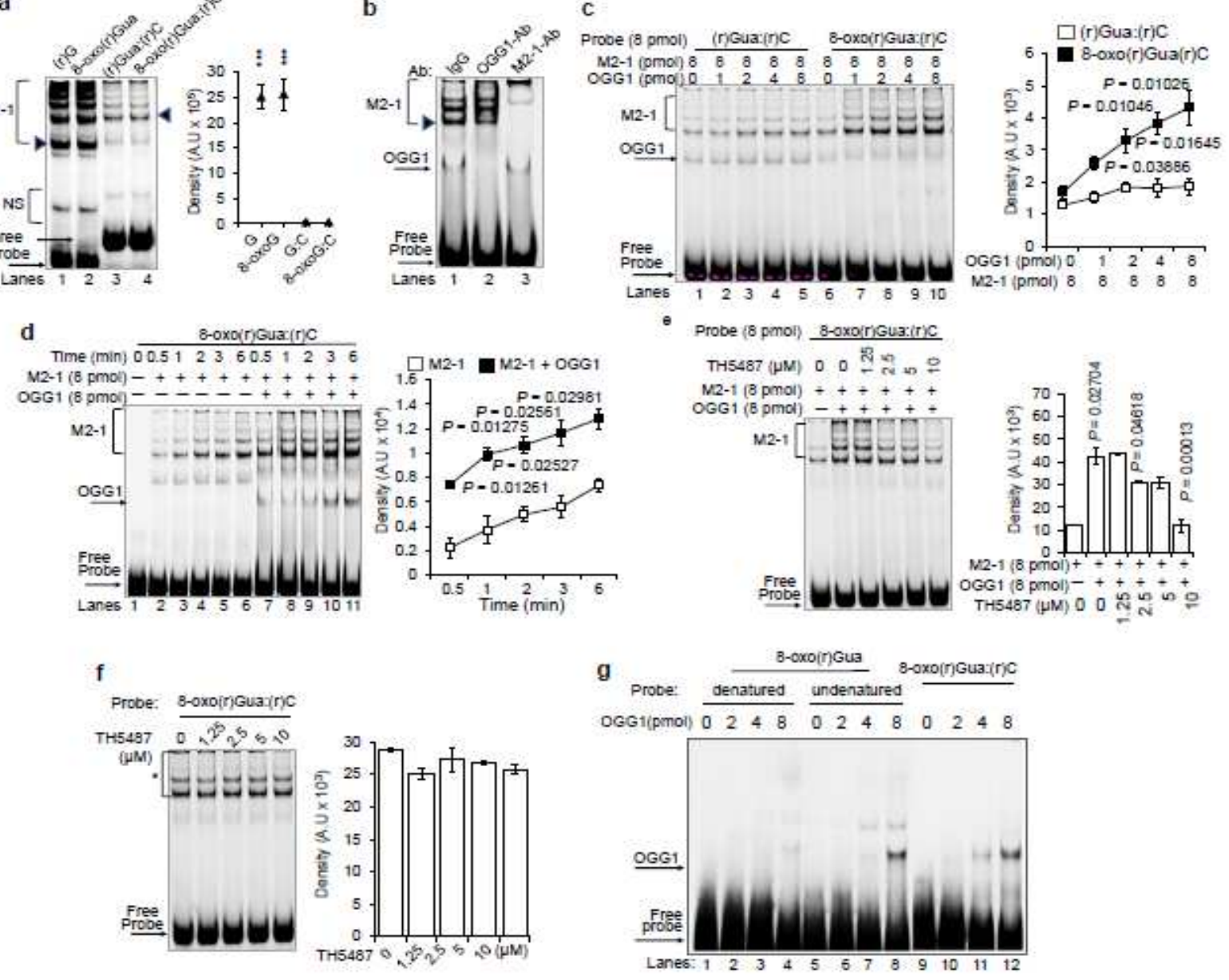

h

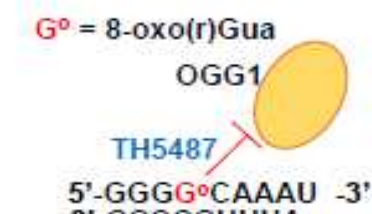

3'-CCCCGUUUA
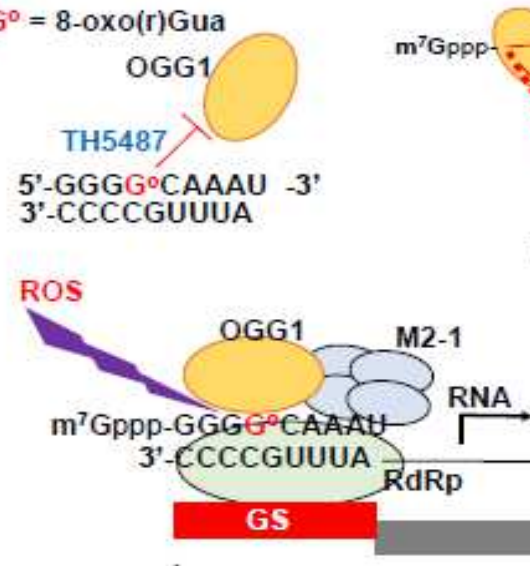

Probe (8 pmoi) 8-oxoirigua:iric

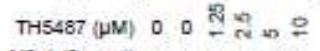
N2-1 (8 pmol) ++++++ OGG1 (8 pmol) -++++++

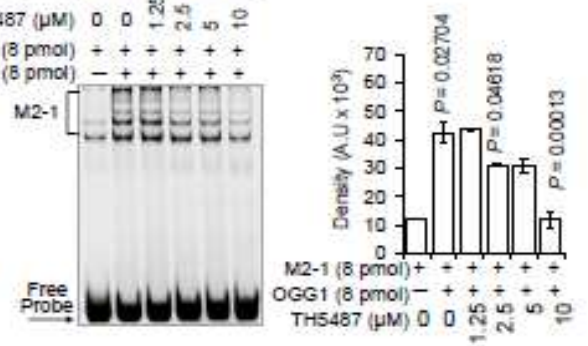

880 OGG1 bound to 5'-UTR of nRNA via 8-oxo(r)Gua facilitates M2-1' interaction with

881 transcriptionally active site

882

a M2-1 lacks ability to recognize 8-oxo(r)Gua in single and double stranded RNAs. Probes \pm 8 oxo(r)Gua were incubated with M2-1, and the magnitude of binding was determined by EMSA. 
Lanes 1, ssRNA: (r)G); 2, ssRNA containing 8-oxo(r)Gua; lane 3, dsRNA [(r)Gua:(r)C; lane 4: dsRNA containing 8-oxo(r)Gua, [8-oxo(r)Gua:(r)C]; arrowhead left and right: M2-1 tetramer-RNA complexes. Right panel, graphical depiction of band intensities ( $n=3$, Image J v1.5.1). ${ }^{* *} p<$ 0.001 .

b Identification of OGG1 and M2-1 8-oxo(r)Gua:(r)C) complexes by super-shift assays. OGG1and M2-1-8-oxo(r)Gua:(r)C complexes were incubated with IgG (lane 1), Ab to OGG1 (lane 2) or Ab to M2-1 (lane 3), and mixtures were subjected to EMSA.

\section{c M2-1 binding to 8-oxo(r)Gua:(r)C is OGG1 concentration dependent. dsRNA probes were} incubated with increasing amounts of $\mathrm{OGG}_{1}$ and then $\mathrm{M} 2-1$ (8 pmol) was added. M2-1 binding in the presence of OGG1 to 8-oxo(r)Gua:(r)C) was determined by EMSA (left panel). Lanes 1 to 5, dsRNA [(r)Gua:(r)C; lane 6 to 10, dsRNA containing 8-oxo(r)Gua [8-oxo(r)Gua:(r)C]. Right panel: band intensities were quantified by Image $J$ v1.51). P values are as in right panel $(n=3)$. d Binding kinetics of M2-1 to 8-oxo(r)Gua:(r)C) \pm OGG1. Equimolar amounts of OGG1, M2-1 and probe [8-oxo(r)Gua:(r)C)] were mixed for various lengths of time ${ }_{2}$ and EMSA was performed. Right panel, graphical depictions of band intensities (Image J v1.51).

e TH5487 decreases OGG1' ability to facilitate M2-1 8-oxo(r)Gua:(r)C interaction. Increasing concentrations of inhibitor were pre-incubated with OGG1, then 8-oxo(r)Gua:(r)C and M2-1 were added before performing EMSA. Right panel, graphical depiction of M2-1 binding $(n=3)$. f TH5487 alone has no effect on M2-1' binding. Increasing concentrations of TH5487 were incubated with M2-1. Tthen probe [8-oxo(r)Gua:(r)C] was added and EMSA was performed. Right panel, graphical depiction of band intensities.

g OGG1 binds 8-oxo(r)Gua-containing temperature-sensitive secondary structure of viral RNA. OGG1 was incubated with 8-oxo(r)Gua containing ssRNA before and after heat denaturation. Binding was assessed by EMSA. In controls, dsRNA (8-oxo(r)Gua:C) was used. Lane 1 to 4, heat denatured ssRNA; lanes 5 to 8: un-denatured RNA, lane 9-12: 8-oxo(r)Gua:(r)C probe. Right panel, structure of the 40 nt long RNA probe as predicted by "RNAstructure" software ${ }^{59}$. h Schematic diagram showing roles of OGG1 in mRNA synthesis. OGG1 recognizes 8oxo(r)Gua the ROS-generated epitranscriptomic mark in the 5'-UTR of nRNA, while paired with the 3 '-end of the gene and functions as a nucleation site for binding of the transcriptional elongation factor M2-1. OGG1 remain bound to the secondary structure of mRNA (Fig. g). Structure of 5'-end of G-mRNA as predicted as in " $g$ ". Secondary structure of G-mRNA is shown Supplementary Fig. 4i. OGG1 agonist (TH5487) inhibits binding of OGG1 to 8-oxo(r)Gua in RNA-RNA hybrid. Lower panel: G-gene is shown. GS, gene start (red) and GE, gene end 
(green). OGG1, 8-oxoguanine DNA glycosylase1; TH5487, active site inhibitor of OGG149. RdRp, RNA dependent RNA polymerase, m7Gppp, methyl guanosine cap.

1 Hall, C. B. et al. The burden of respiratory syncytial virus infection in young children. $N$

923

924 Engl J Med 360, 588-598, doi:10.1056/NEJMoa0804877 (2009).

925

926

927

928

929

930 syncytial virus in young children: a systematic review and meta-analysis. Lancet $\mathbf{3 7 5}$, 1545-1555, doi:10.1016/S0140-6736(10)60206-1 (2010).

3 Aherne, W., Bird, T., Court, S. D., Gardner, P. S. \& McQuillin, J. Pathological changes in virus infections of the lower respiratory tract in children. J Clin Pathol 23, 7-18, doi:10.1136/jcp.23.1.7 (1970).

4 Falsey, A. R., Hennessey, P. A., Formica, M. A., Cox, C. \& Walsh, E. E. Respiratory syncytial virus infection in elderly and high-risk adults. $N$ Engl J Med 352, 1749-1759, doi:10.1056/NEJMoa043951 (2005).

5 Lee, N. et al. High morbidity and mortality in adults hospitalized for respiratory syncytial virus infections. Clin Infect Dis 57, 1069-1077, doi:10.1093/cid/cit471 (2013).

$6 \quad$ Welliver, T. P. et al. Severe human lower respiratory tract illness caused by respiratory syncytial virus and influenza virus is characterized by the absence of pulmonary cytotoxic lymphocyte responses. J Infect Dis 195, 1126-1136, doi:10.1086/512615 (2007).

7 Mazur, N. I. et al. The respiratory syncytial virus vaccine landscape: lessons from the graveyard and promising candidates. Lancet Infect Dis 18, e295-e311, doi:10.1016/S1473-3099(18)30292-5 (2018).

8 Tayyari, F. et al. Identification of nucleolin as a cellular receptor for human respiratory syncytial virus. Nat Med 17, 1132-1135, doi:10.1038/nm.2444 (2011).

9 Krzyzaniak, M. A., Zumstein, M. T., Gerez, J. A., Picotti, P. \& Helenius, A. Host cell entry of respiratory syncytial virus involves macropinocytosis followed by proteolytic activation of the F protein. PLoS Pathog 9, e1003309, doi:10.1371/journal.ppat.1003309 (2013).

10 Noton, S. L., Tremaglio, C. Z. \& Fearns, R. Killing two birds with one stone: How the respiratory syncytial virus polymerase initiates transcription and replication. PLoS Pathog 15, e1007548, doi:10.1371/journal.ppat.1007548 (2019).

11 Braun, M. R. et al. RNA elongation by respiratory syncytial virus polymerase is calibrated by conserved region V. PLoS Pathog 13, e1006803, doi:10.1371/journal.ppat.1006803 (2017).

12 Cressey, T. N., Noton, S. L., Nagendra, K., Braun, M. R. \& Fearns, R. Mechanism for de novo initiation at two sites in the respiratory syncytial virus promoter. Nucleic Acids Res 46, 6785-6796, doi:10.1093/nar/gky480 (2018).

13 Kuo, L., Grosfeld, H., Cristina, J., Hill, M. G. \& Collins, P. L. Effects of mutations in the gene-start and gene-end sequence motifs on transcription of monocistronic and dicistronic minigenomes of respiratory syncytial virus. J Virol 70, 6892-6901, doi:10.1128/JVI.70.10.6892-6901.1996 (1996).

14 Noton, S. L., Deflube, L. R., Tremaglio, C. Z. \& Fearns, R. The respiratory syncytial virus polymerase has multiple RNA synthesis activities at the promoter. PLoS Pathog 8 , e1002980, doi:10.1371/journal.ppat.1002980 (2012). 
1000

1001

1002

1003

1004

1005

1006

1007

1008

1009

1010

1011

15 Kleiner, V. A. \& Fearns, R. RSV M2-1 Protein in Complex with RNA: Old Questions Are Answered and a New One Emerges. Structure 28, 977-978, doi:10.1016/j.str.2020.08.007 (2020).

16 Fricke, J., Koo, L. Y., Brown, C. R. \& Collins, P. L. p38 and OGT sequestration into viral inclusion bodies in cells infected with human respiratory syncytial virus suppresses MK2 activities and stress granule assembly. J Virol 87, 1333-1347, doi:10.1128/JVI.02263-12 (2013).

17 Collins, P. L. \& Graham, B. S. Viral and host factors in human respiratory syncytial virus pathogenesis. J Virol 82, 2040-2055, doi:10.1128/JVI.01625-07 (2008).

18 Brasier, A. R. et al. Nuclear heat shock response and novel nuclear domain 10 reorganization in respiratory syncytial virus-infected a549 cells identified by highresolution two-dimensional gel electrophoresis. J Virol 78, 11461-11476, doi:10.1128/JVI.78.21.11461-11476.2004 (2004).

19 Kipper, S. et al. New host factors important for respiratory syncytial virus (RSV) replication revealed by a novel microfluidics screen for interactors of matrix (M) protein. Mol Cell Proteomics 14, 532-543, doi:10.1074/mcp.M114.044107 (2015).

20 Mehedi, M. et al. Actin-Related Protein 2 (ARP2) and Virus-Induced Filopodia Facilitate Human Respiratory Syncytial Virus Spread. PLoS Pathog 12, e1006062, doi:10.1371/journal.ppat.1006062 (2016).

21 Tsai, K. \& Cullen, B. R. Epigenetic and epitranscriptomic regulation of viral replication. Nat Rev Microbiol 18, 559-570, doi:10.1038/s41579-020-0382-3 (2020).

22 Pereira-Montecinos, C., Valiente-Echeverria, F. \& Soto-Rifo, R. Epitranscriptomic regulation of viral replication. Biochim Biophys Acta Gene Regul Mech 1860, 460-471, doi:10.1016/j.bbagrm.2017.02.002 (2017).

23 Song, J. \& Yi, C. Chemical Modifications to RNA: A New Layer of Gene Expression Regulation. ACS Chem Biol 12, 316-325, doi:10.1021/acschembio.6b00960 (2017).

24 Netzband, R. \& Pager, C. T. Epitranscriptomic marks: Emerging modulators of RNA virus gene expression. Wiley Interdiscip Rev RNA 11, e1576, doi:10.1002/wrna.1576 (2020).

25 Gokhale, N. S. \& Horner, S. M. RNA modifications go viral. PLoS Pathog 13, e1006188, doi:10.1371/journal.ppat.1006188 (2017).

26 Balakrishnan, L. \& Milavetz, B. Epigenetic Regulation of Viral Biological Processes. Viruses 9, doi:10.3390/v9110346 (2017).

27 Hofer, T. et al. Hydrogen peroxide causes greater oxidation in cellular RNA than in DNA. Biol Chem 386, 333-337, doi:10.1515/BC.2005.040 (2005).

28 Radak, Z. \& Boldogh, I. 8-Oxo-7,8-dihydroguanine: links to gene expression, aging, and defense against oxidative stress. Free Radic Biol Med 49, 587-596, doi:10.1016/j.freeradbiomed.2010.05.008 (2010).

29 Kong, Q. \& Lin, C. L. Oxidative damage to RNA: mechanisms, consequences, and diseases. Cell Mol Life Sci 67, 1817-1829, doi:10.1007/s00018-010-0277-y (2010).

30 Pratviel, G. \& Meunier, B. Guanine oxidation: one- and two-electron reactions. Chemistry 12, 6018-6030, doi:10.1002/chem.200600539 (2006).

31 Simms, C. L. \& Zaher, H. S. Quality control of chemically damaged RNA. Cell Mol Life Sci 73, 3639-3653, doi:10.1007/s00018-016-2261-7 (2016).

32 Mitra, S. et al. Complexities of DNA base excision repair in mammalian cells. Mol Cells 7, 305-312 (1997).

33 Ishii, T. \& Sekiguchi, M. Two ways of escaping from oxidative RNA damage: Selective degradation and cell death. DNA Repair (Amst) 81, 102666, doi:10.1016/j.dnarep.2019.102666 (2019). 
$101234 \quad$ Hosakote, Y. M. et al. Antioxidant mimetics modulate oxidative stress and cellular

1013

1014

1015

1016

1017

1018

1019

1020

1021

1022

1023

1024

1025

1026

1027

1028

1029

1030

1031

1032

1033

1034

1035

1036

1037

1038

1039

1040

1041

1042

1043

1044

1045

1046

1047

1048

1049

1050

1051

1052

1053

1054

1055

1056

1057

1058

1059

1060

1061

1062 signaling in airway epithelial cells infected with respiratory syncytial virus. Am J Physiol Lung Cell Mol Physiol 303, L991-1000 (2012).

35 Ivanciuc, T., Sbrana, E., Casola, A. \& Garofalo, R. P. Protective Role of Nuclear Factor Erythroid 2-Related Factor 2 Against Respiratory Syncytial Virus and Human Metapneumovirus Infections. Front Immuno/ 9, 854, doi:10.3389/fimmu.2018.00854 (2018).

36 Garofalo, R. P., Kolli, D. \& Casola, A. Respiratory syncytial virus infection: mechanisms of redox control and novel therapeutic opportunities. Antioxid Redox Signal 18, 186-217, doi:10.1089/ars.2011.4307 (2013).

37 Khomich, O. A., Kochetkov, S. N., Bartosch, B. \& Ivanov, A. V. Redox Biology of Respiratory Viral Infections. Viruses 10, doi:10.3390/v10080392 (2018).

38 D'Augustin, O., Huet, S., Campalans, A. \& Radicella, J. P. Lost in the Crowd: How Does Human 8-Oxoguanine DNA Glycosylase 1 (OGG1) Find 8-Oxoguanine in the Genome? Int J Mol Sci 21, doi:10.3390/ijms21218360 (2020).

39 Zhao, Y. et al. Systematic Analysis of Cell-Type Differences in the Epithelial Secretome Reveals Insights into the Pathogenesis of Respiratory Syncytial Virus-Induced Lower Respiratory Tract Infections. J Immunol 198, 3345-3364, doi:10.4049/jimmunol.1601291 (2017).

40 Tian, B. et al. Central Role of the NF-kappaB Pathway in the Scgb1a1-Expressing Epithelium in Mediating Respiratory Syncytial Virus-Induced Airway Inflammation. J Virol 92, doi:10.1128/JVI.00441-18 (2018).

41 Amente, S. et al. Genome-wide mapping of 8-oxo-7,8-dihydro-2'-deoxyguanosine reveals accumulation of oxidatively-generated damage at DNA replication origins within transcribed long genes of mammalian cells. Nucleic Acids Res 47, 221-236, doi:10.1093/nar/gky1152 (2019).

42 de Jager, T. L., Cockrell, A. E. \& Du Plessis, S. S. Ultraviolet Light Induced Generation of Reactive Oxygen Species. Adv Exp Med Bio/ 996, 15-23, doi:10.1007/978-3-31956017-5_2 (2017).

43 Noton, S. L. \& Fearns, R. Initiation and regulation of paramyxovirus transcription and replication. Virology 479-480, 545-554, doi:10.1016/j.virol.2015.01.014 (2015).

44 Tran, T. L. et al. The respiratory syncytial virus M2-1 protein forms tetramers and interacts with RNA and P in a competitive manner. $J$ Virol 83, 6363-6374, doi:10.1128/JVI.00335-09 (2009).

45 Nakabeppu, Y., Ohta, E. \& Abolhassani, N. MTH1 as a nucleotide pool sanitizing enzyme: Friend or foe? Free Radic Biol Med 107, 151-158, doi:10.1016/j.freeradbiomed.2016.11.002 (2017).

46 Tornaletti, S., Maeda, L. S., Kolodner, R. D. \& Hanawalt, P. C. Effect of 8-oxoguanine on transcription elongation by T7 RNA polymerase and mammalian RNA polymerase II. DNA Repair (Amst) 3, 483-494, doi:10.1016/j.dnarep.2004.01.003 (2004).

47 Zhang, Y. et al. Ribavirin treatment up-regulates antiviral gene expression via the interferon-stimulated response element in respiratory syncytial virus-infected epithelial cells. J Virol 77, 5933-5947, doi:10.1128/jvi.77.10.5933-5947.2003 (2003).

48 Hazra, T. K. et al. Identification and characterization of a novel human DNA glycosylase for repair of cytosine-derived lesions. J Biol Chem 277, 30417-30420, doi:10.1074/jbc.C200355200 (2002).

49 Visnes, T. et al. Small-molecule inhibitor of OGG1 suppresses proinflammatory gene expression and inflammation. Science 362, 834-839, doi:10.1126/science.aar8048 (2018).

50 Donley, N. et al. Small Molecule Inhibitors of 8-Oxoguanine DNA Glycosylase-1 (OGG1). ACS Chem Biol 10, 2334-2343, doi:10.1021/acschembio.5b00452 (2015). 
$106351 \quad$ Hall, D. B., Holmlin, R. E. \& Barton, J. K. Oxidative DNA damage through long-range

1064

1065 electron transfer. Nature 382, 731-735, doi:10.1038/382731a0 (1996).

1066

1067

1068

1069

1070

1071

1072

1073

1074

1075

1076

1077

1078

1079

1080

1081

1082

1083

1084

1085

1086

1087

1088

1089

1090

1091

1092

1093

1094

1095

1096

1097

1098

1099

1100

1101

1102

1103

1104

1105

1106

1107

1108

1109

52 Tekes, G., Rahmeh, A. A. \& Whelan, S. P. A freeze frame view of vesicular stomatitis virus transcription defines a minimal length of RNA for 5' processing. PLoS Pathog 7, e1002073, doi:10.1371/journal.ppat.1002073 (2011).

$53 \mathrm{Ba}, \mathrm{X}$. et al. 8-oxoguanine DNA glycosylase-1 augments proinflammatory gene expression by facilitating the recruitment of site-specific transcription factors. $J$ Immunol 192, 2384-2394, doi:10.4049/jimmunol.1302472 (2014).

54 Tanner, S. J. et al. Crystal structure of the essential transcription antiterminator M2-1 protein of human respiratory syncytial virus and implications of its phosphorylation. Proc Natl Acad Sci U S A 111, 1580-1585, doi:10.1073/pnas.1317262111 (2014).

55 Rincheval, V. et al. Functional organization of cytoplasmic inclusion bodies in cells infected by respiratory syncytial virus. Nat Commun 8, 563, doi:10.1038/s41467-01700655-9 (2017).

56 Hegde, P. M. et al. The C-terminal Domain (CTD) of Human DNA Glycosylase NEIL1 Is Required for Forming BERosome Repair Complex with DNA Replication Proteins at the Replicating Genome: DOMINANT NEGATIVE FUNCTION OF THE CTD. J Biol Chem 290, 20919-20933, doi:10.1074/jbc.M115.642918 (2015).

57 Dunn, K. W., Kamocka, M. M. \& McDonald, J. H. A practical guide to evaluating colocalization in biological microscopy. Am J Physiol Cell Physiol 300, C723-742, doi:10.1152/ajpcell.00462.2010 (2011).

58 Esperante, S. A. et al. Fine modulation of the respiratory syncytial virus M2-1 protein quaternary structure by reversible zinc removal from its Cys(3)-His(1) motif. Biochemistry 52, 6779-6789, doi:10.1021/bi401029q (2013).

59 Zhou, Y. \& Routh, A. Mapping RNA-capsid interactions and RNA secondary structure within virus particles using next-generation sequencing. Nucleic Acids Res 48, e12, doi:10.1093/nar/gkz1124 (2020).

60 Gao, Y. et al. Structure of the Human Respiratory Syncytial Virus M2-1 Protein in Complex with a Short Positive-Sense Gene-End RNA. Structure 28, 979-990 e974, doi:10.1016/j.str.2020.07.001 (2020).

61 Bruner, S. D., Norman, D. P. \& Verdine, G. L. Structural basis for recognition and repair of the endogenous mutagen 8-oxoguanine in DNA. Nature 403, 859-866, doi:10.1038/35002510 (2000).

62 Bakker, S. E. et al. The respiratory syncytial virus nucleoprotein-RNA complex forms a left-handed helical nucleocapsid. J Gen Virol 94, 1734-1738, doi:10.1099/vir.0.053025-0 (2013).

63 Steenken, S. Structure, acid/base properties and transformation reactions of purine radicals. Free Radic Res Commun 6, 117-120, doi:10.3109/10715768909073445 (1989).

$64 \mathrm{Ba}, \mathrm{X}$. \& Boldogh, I. 8-Oxoguanine DNA glycosylase 1: Beyond repair of the oxidatively modified base lesions. Redox Biol 14, 669-678, doi:10.1016/j.redox.2017.11.008 (2018).

65 Brasier, A. R. \& Boldogh, I. Targeting inducible epigenetic reprogramming pathways in chronic airway remodeling. Drugs Context 8, doi:10.7573/dic.2019-8-3 (2019).

66 Blondot, M. L. et al. Structure and functional analysis of the RNA- and viral phosphoprotein-binding domain of respiratory syncytial virus M2-1 protein. PLoS Pathog 8, e1002734, doi:10.1371/journal.ppat.1002734 (2012).

67 Di Giorgio, S., Martignano, F., Torcia, M. G., Mattiuz, G. \& Conticello, S. G. Evidence for host-dependent RNA editing in the transcriptome of SARS-CoV-2. Sci Adv 6, eabb5813, doi:10.1126/sciadv.abb5813 (2020). 
111268 Xue, M. et al. Viral N(6)-methyladenosine upregulates replication and pathogenesis of human respiratory syncytial virus. Nat Commun 10, 4595, doi:10.1038/s41467-01912504-y (2019).

69 Jaafar, Z. A. \& Kieft, J. S. Viral RNA structure-based strategies to manipulate translation. Nat Rev Microbiol 17, 110-123, doi:10.1038/s41579-018-0117-x (2019).

70 Saxowsky, T. T., Meadows, K. L., Klungland, A. \& Doetsch, P. W. 8-Oxoguaninemediated transcriptional mutagenesis causes Ras activation in mammalian cells. Proc Natl Acad Sci U S A 105, 18877-18882, doi:10.1073/pnas.0806464105 (2008).

71 Castro, C., Arnold, J. J. \& Cameron, C. E. Incorporation fidelity of the viral RNAdependent RNA polymerase: a kinetic, thermodynamic and structural perspective. Virus Res 107, 141-149, doi:10.1016/j.virusres.2004.11.004 (2005).

72 Hause, A. M. et al. Sequence variability of the respiratory syncytial virus (RSV) fusion gene among contemporary and historical genotypes of RSV/A and RSV/B. PLoS One 12, e0175792, doi:10.1371/journal.pone.0175792 (2017).

73 Patel, J. A. et al. Interleukin-1 alpha mediates the enhanced expression of intercellular adhesion molecule- 1 in pulmonary epithelial cells infected with respiratory syncytial

virus. Am J Respir Cell Mol Biol 13, 602-609, doi:10.1165/ajrcmb.13.5.7576697 (1995).

74 Hao, W. et al. Effects of the stimuli-dependent enrichment of 8-oxoguanine DNA glycosylase 1 on chromatinized DNA. Redox Biol 18, 43-53, doi:10.1016/j.redox.2018.06.002 (2018). 
1134 


\section{Supplementary Files}

This is a list of supplementary files associated with this preprint. Click to download.

- Wangetal.Supplymentary.pdf 